\title{
Indefinite Affine Hyperspheres Admitting a Pointwise Symmetry. Part $2^{\star}$
}

\author{
Christine SCHARLACH
}

Technische Universität Berlin, Fak. II, Inst. f. Mathematik, MA 8-3, 10623 Berlin, Germany

E-mail:schar@math.tu-berlin.de

URL: http://www.math.tu-berlin.de/ schar/

Received May 08, 2009, in final form October 06, 2009; Published online October 19, 2009

doi:10.3842/SIGMA.2009.097

\begin{abstract}
An affine hypersurface $M$ is said to admit a pointwise symmetry, if there exists a subgroup $G$ of $\operatorname{Aut}\left(T_{p} M\right)$ for all $p \in M$, which preserves (pointwise) the affine metric $h$, the difference tensor $K$ and the affine shape operator $S$. Here, we consider 3-dimensional indefinite affine hyperspheres, i.e. $S=H I d$ (and thus $S$ is trivially preserved). In Part 1 we found the possible symmetry groups $G$ and gave for each $G$ a canonical form of $K$. We started a classification by showing that hyperspheres admitting a pointwise $\mathbb{Z}_{2} \times \mathbb{Z}_{2}$ resp. $\mathbb{R}$ symmetry are well-known, they have constant sectional curvature and Pick invariant $J<0$ resp. $J=0$. Here, we continue with affine hyperspheres admitting a pointwise $\mathbb{Z}_{3^{-}}$or $S O(2)$-symmetry. They turn out to be warped products of affine spheres $\left(\mathbb{Z}_{3}\right)$ or quadrics $(S O(2))$ with a curve.
\end{abstract}

Key words: affine hyperspheres; indefinite affine metric; pointwise symmetry; affine differential geometry; affine spheres; warped products

2000 Mathematics Subject Classification: 53A15; 53B30

\section{Introduction}

Let $M^{n}$ be a connected, oriented manifold. Consider an immersed hypersurface with relative normalization, i.e., an immersion $\varphi: M^{n} \rightarrow \mathbb{R}^{n+1}$ together with a transverse vector field $\xi$ such that $D \xi$ has its image in $\varphi_{*} T_{x} M$. Equi-affine geometry studies the properties of such immersions under equi-affine transformations, i.e. volume-preserving linear transformations $(S L(n+1, \mathbb{R}))$ and translations.

In the theory of nondegenerate equi-affine hypersurfaces there exists a canonical choice of transverse vector field $\xi$ (unique up to sign), called the affine (Blaschke) normal, which induces a connection $\nabla$, a nondegenerate symmetric bilinear form $h$ and a 1-1 tensor field $S$ by

$$
\begin{aligned}
& D_{X} Y=\nabla_{X} Y+h(X, Y) \xi, \\
& D_{X} \xi=-S X
\end{aligned}
$$

for all $X, Y \in \mathcal{X}(M)$. The connection $\nabla$ is called the induced affine connection, $h$ is called the affine metric or Blaschke metric and $S$ is called the affine shape operator. In general $\nabla$ is not the Levi-Civita connection $\hat{\nabla}$ of $h$. The difference tensor $K$ is defined as

$$
K(X, Y)=\nabla_{X} Y-\hat{\nabla}_{X} Y
$$

for all $X, Y \in \mathcal{X}(M)$. Moreover the form $h(K(X, Y), Z)$ is a symmetric cubic form with the property that for any fixed $X \in \mathcal{X}(M)$, trace $K_{X}$ vanishes. This last property is called the

\footnotetext{
*This paper is a contribution to the Special Issue "Élie Cartan and Differential Geometry". The full collection is available at http://www.emis.de/journals/SIGMA/Cartan.html
} 
apolarity condition. The difference tensor $K$, together with the affine metric $h$ and the affine shape operator $S$ are the most fundamental algebraic invariants for a nondegenerate affine hypersurface (more details in Section 2). We say that $M^{n}$ is indefinite, definite, etc. if the affine metric $h$ is indefinite, definite, etc. (Because the affine metric is a multiple of the Euclidean second fundamental form, a positive definite hypersurface is locally strongly convex.) For details of the basic theory of nondegenerate affine hypersurfaces we refer to [7] and [10].

Here we will restrict ourselves to the case of affine hyperspheres, i.e. the shape operator will be a (constant) multiple of the identity $(S=H I d)$. Geometrically this means that all affine normals pass through a fixed point or they are parallel. There are many affine hyperspheres, even in the two-dimensional case only partial classifications are known. This is due to the fact that affine hyperspheres reduce to the study of the Monge-Ampère equations. Our question is the following: What can we say about a three-dimensional affine hypersphere which admits a pointwise $G$-symmetry, i.e. there exists a non-trivial subgroup $G$ of the isometry group such that for every $p \in M$ and every $L \in G$ :

$$
K\left(L X_{p}, L Y_{p}\right)=L\left(K\left(X_{p}, Y_{p}\right)\right) \quad \forall X_{p}, Y_{p} \in T_{p} M .
$$

We have motivated this question in Part 1 [13] (see also [1, 14, 8]). A classification of 3-dimensional positive definite affine hyperspheres admitting pointwise symmetries was obtained in [14]. We continue the classification in the indefinite case. We can assume that the affine metric has index two, i.e. the corresponding isometry group is the (special) Lorentz group $\mathrm{SO}(1,2)$. In Part 1, it turns out that a $\mathrm{SO}(1,2)$-stabilizer of a nontrivial traceless cubic form is isomorphic to either $\mathrm{SO}(2), \mathrm{SO}(1,1), \mathbb{R}$, the group $S_{3}$ of order $6, \mathbb{Z}_{2} \times \mathbb{Z}_{2}, \mathbb{Z}_{3}, \mathbb{Z}_{2}$ or it is trivial. We have shown that hyperspheres admitting a pointwise $\mathbb{Z}_{2} \times \mathbb{Z}_{2}$ - resp. $\mathbb{R}$-symmetry are well-known, they have constant sectional curvature and Pick invariant $J<0$ resp. $J=0$.

In the following we classify the indefinite affine hyperspheres which admit a pointwise $\mathbb{Z}_{3^{-}}$, $\mathrm{SO}(2)$ - or $\mathrm{SO}(1,1)$-symmetry. They turn out to be warped products of affine spheres $\left(\mathbb{Z}_{3}\right)$ or quadrics $(S O(2), S O(1,1))$ with a curve. As a result we get a new composition method. Since the methods for the proofs for $S O(1,1)$ are similar to those for $\mathbb{Z}_{3^{-}}$or $\mathrm{SO}(2)$-symmetry (and as long) we will omit them here. Both methods and results are different in case of $S_{3}$-symmetry and will be published elsewhere. The paper is organized as follows:

We will state the basic formulas of (equi-)affine hypersurface-theory needed in the further classification in Section 2. In Section 3, we show that in case of $\mathrm{SO}(2)$-, $S_{3}$ - or $\mathbb{Z}_{3}$-symmetry we can extend the canonical form of $K$ (cf. [13]) locally. Thus we can obtain information about the coefficients of $K$ and $\nabla$ from the basic equations of Gauss, Codazzi and Ricci (cf. Section 4). In Section 5 we show, that in case of $\mathbb{Z}_{3^{-}}$or $\mathrm{SO}(2)$-symmetry it follows that the hypersurface admits a warped product structure $\mathbb{R} \times_{e^{f}} N^{2}$. Then we classify such hyperspheres by showing how they can be constructed starting from 2-dimensional positive definite affine spheres resp. quadrics (cf. Theorems 1-8). We end in Section 6 by stating the classification results in case of $S O(1,1)$-symmetry (cf. Theorems 9-16).

The classification can be seen as a generalization of the well known Calabi product of hyperbolic affine spheres $[2,6]$ and of the constructions for affine spheres considered in [5]. The following natural question for a (de)composition theorem, related to these constructions, gives another motivation for studying 3-dimensional hypersurfaces admitting a pointwise symmetry:

(De)composition Problem. Let $M^{n}$ be a nondegenerate affine hypersurface in $\mathbb{R}^{n+1}$. Under what conditions do there exist affine hyperpsheres $M_{1}^{r}$ in $\mathbb{R}^{r+1}$ and $M_{2}^{s}$ in $\mathbb{R}^{s+1}$, with $r+s=n-1$, such that $M=I \times{ }_{f_{1}} M_{1} \times_{f_{2}} M_{2}$, where $I \subset \mathbb{R}$ and $f_{1}$ and $f_{2}$ depend only on $I$ (i.e. $M$ admits a warped product structure)? How can the original immersion be recovered starting from the immersion of the affine spheres?

Of course the first dimension in which the above problem can be considered is three and our results provide an answer in that case. 


\section{Basics of affine hypersphere theory}

First we recall the definition of the affine normal $\xi$ (cf. [10]). In equi-affine hypersurface theory on the ambient space $\mathbb{R}^{n+1}$ a fixed volume form det is given. A transverse vector field $\xi$ induces a volume form $\theta$ on $M$ by $\theta\left(X_{1}, \ldots, X_{n}\right)=\operatorname{det}\left(\varphi_{*} X_{1}, \ldots, \varphi_{*} X_{n}, \xi\right)$. Also the affine metric $h$ defines a volume form $\omega_{h}$ on $M$, namely $\omega_{h}=|\operatorname{det} h|^{1 / 2}$. Now the affine normal $\xi$ is uniquely determined (up to sign) by the conditions that $D \xi$ is everywhere tangential (which is equivalent to $\nabla \theta=0)$ and that

$$
\theta=\omega_{h}
$$

Since we only consider 3-dimensional indefinite hyperspheres, i.e.

$$
S=H I d, \quad H=\text { const }
$$

we can fix the orientation of the affine normal $\xi$ such that the affine metric has signature one. Then the sign of $H$ in the definition of an affine hypersphere is an invariant.

Next we state some of the fundamental equations, which a nondegenerate hypersurface has to satisfy, see also [10] or [7]. These equations relate $S$ and $K$ with amongst others the curvature tensor $R$ of the induced connection $\nabla$ and the curvature tensor $\hat{R}$ of the Levi-Civita connection $\widehat{\nabla}$ of the affine metric $h$. There are the Gauss equation for $\nabla$, which states that:

$$
R(X, Y) Z=h(Y, Z) S X-h(X, Z) S Y,
$$

and the Codazzi equation

$$
\left(\nabla_{X} S\right) Y=\left(\nabla_{Y} S\right) X
$$

Also we have the total symmetry of the affine cubic form

$$
C(X, Y, Z)=\left(\nabla_{X} h\right)(Y, Z)=-2 h(K(X, Y), Z) .
$$

The fundamental existence and uniqueness theorem, see [3] or [4], states that given $h, \nabla$ and $S$ such that the difference tensor is symmetric and traceless with respect to $h$, on a simply connected manifold $M$ an affine immersion of $M$ exists if and only if the above Gauss equation and Codazzi equation are satisfied.

From the Gauss equation and Codazzi equation above the Codazzi equation for $K$ and the Gauss equation for $\widehat{\nabla}$ follow:

$$
\left(\widehat{\nabla}_{X} K\right)(Y, Z)-\left(\widehat{\nabla}_{Y} K\right)(X, Z)=\frac{1}{2}(h(Y, Z) S X-h(X, Z) S Y-h(S Y, Z) X+h(S X, Z) Y),
$$

and

$$
\hat{R}(X, Y) Z=\frac{1}{2}(h(Y, Z) S X-h(X, Z) S Y+h(S Y, Z) X-h(S X, Z) Y)-\left[K_{X}, K_{Y}\right] Z
$$

If we define the Ricci tensor of the Levi-Civita connection $\widehat{\nabla}$ by:

$$
\widehat{\operatorname{Ric}}(X, Y)=\operatorname{trace}\{Z \mapsto \hat{R}(Z, X) Y\} \text {. }
$$

and the Pick invariant by:

$$
J=\frac{1}{n(n-1)} h(K, K),
$$


then from the Gauss equation we get for the scalar curvature $\hat{\kappa}=\frac{1}{n(n-1)}\left(\sum_{i, j} h^{i j} \widehat{\operatorname{Ric}}_{i j}\right)$ :

$$
\hat{\kappa}=H+J .
$$

For an affine hypersphere the Gauss and Codazzi equations have the form:

$$
\begin{aligned}
& R(X, Y) Z=H(h(Y, Z) X-h(X, Z) Y), \\
& \left(\nabla_{X} H\right) Y=\left(\nabla_{Y} H\right) X, \quad \text { i.e. } \quad H=\text { const, } \\
& \left(\widehat{\nabla}_{X} K\right)(Y, Z)=\left(\widehat{\nabla}_{Y} K\right)(X, Z), \\
& \hat{R}(X, Y) Z=H(h(Y, Z) X-h(X, Z) Y)-\left[K_{X}, K_{Y}\right] Z .
\end{aligned}
$$

Since $H$ is constant, we can rescale $\varphi$ such that $H \in\{-1,0,1\}$.

\section{A local frame for pointwise $\mathrm{SO}(2)-, S_{3^{-}}$or $\mathbb{Z}_{3^{-} \text {-symmetry }}$}

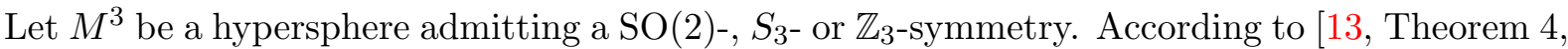
2.-4.] there exists for every $p \in M^{3}$ an ONB $\{\mathbf{t}, \mathbf{v}, \mathbf{w}\}$ of $T_{p} M^{3}$ such that

$$
\begin{array}{lll}
K(\mathbf{t}, \mathbf{t})=-2 a_{4} \mathbf{t}, & K(\mathbf{t}, \mathbf{v})=a_{4} \mathbf{v}, & K(\mathbf{t}, \mathbf{w})=a_{4} \mathbf{w} \\
K(\mathbf{v}, \mathbf{v})=-a_{4} \mathbf{t}+a_{6} \mathbf{v}, & K(\mathbf{v}, \mathbf{w})=-a_{6} \mathbf{w}, & K(\mathbf{w}, \mathbf{w})=-a_{4} \mathbf{t}-a_{6} \mathbf{v}
\end{array}
$$

where $a_{4}>0$ and $a_{6}=0$ in case of $S O(2)$-symmetry, $a_{4}=0$ and $a_{6}>0$ for $S_{3}$, and $a_{4}>0$ and $a_{6}>0$ for $\mathbb{Z}_{3}$.

We would like to extend the ONB locally. It is well known that $\widehat{\operatorname{Ric}}$ (cf. (7)) is a symmetric operator and we compute (some of the computations in this section are done with the CAS Mathematica $\left.^{1}\right)$ :

Lemma 1. Let $p \in M^{3}$ and $\{\mathbf{t}, \mathbf{v}, \mathbf{w}\}$ the basis constructed earlier. Then

$$
\begin{array}{ll}
\widehat{\operatorname{Ric}}(\mathbf{t}, \mathbf{t})=-2\left(H-3 a_{4}^{2}\right), & \widehat{\operatorname{Ric}}(\mathbf{t}, \mathbf{v})=0, \\
\widehat{\operatorname{Ric}}(\mathbf{t}, \mathbf{w})=0, & \widehat{\operatorname{Ric}}(\mathbf{v}, \mathbf{v})=2\left(H-a_{4}^{2}+a_{6}^{2}\right), \\
\widehat{\operatorname{Ric}}(\mathbf{v}, \mathbf{w})=0, & \widehat{\operatorname{Ric}}(\mathbf{w}, \mathbf{w})=2\left(H-a_{4}^{2}+a_{6}^{2}\right) .
\end{array}
$$

Proof. The proof is a straight-forward computation using the Gauss equation (13). It follows e.g. that

$$
\begin{aligned}
& \hat{R}(\mathbf{t}, \mathbf{v}) \mathbf{t}=H \mathbf{v}-K_{\mathbf{t}}\left(a_{4} \mathbf{v}\right)+K_{\mathbf{v}}\left(-2 a_{4} \mathbf{t}\right)=H \mathbf{v}-a_{4}^{2} \mathbf{v}-2 a_{4}^{2} \mathbf{v}=\left(H-3 a_{4}^{2}\right) \mathbf{v}, \\
& \hat{R}(\mathbf{t}, \mathbf{w}) \mathbf{t}=H \mathbf{w}-K_{\mathbf{t}}\left(a_{4} \mathbf{w}\right)+K_{\mathbf{w}}\left(-2 a_{4} \mathbf{t}\right)=H \mathbf{w}-a_{4}^{2} \mathbf{w}-2 a_{4}^{2} \mathbf{w}=\left(H-3 a_{4}^{2}\right) \mathbf{w}, \\
& \hat{R}(\mathbf{t}, \mathbf{v}) \mathbf{w}=-K_{\mathbf{t}}\left(-a_{6} \mathbf{w}\right)+K_{\mathbf{v}}\left(a_{4} \mathbf{w}\right)=0 .
\end{aligned}
$$

From this it immediately follows that

$$
\widehat{\operatorname{Ric}}(\mathbf{t}, \mathbf{t})=-2\left(H-3 a_{4}^{2}\right)
$$

and

$$
\widehat{\operatorname{Ric}}(\mathbf{t}, \mathbf{w})=0 \text {. }
$$

The other equations follow by similar computations.

\footnotetext{
${ }^{1}$ See Appendix or http://www.math.tu-berlin.de/ $\sim$ schar/IndefSym_typ234.html.
} 
We want to show that the basis we have constructed, at each point $p$, can be extended differentiably to a neighborhood of the point $p$ such that, at every point, $K$ with respect to the frame $\{T, V, W\}$ has the previously described form.

Lemma 2. Let $M^{3}$ be an affine hypersphere in $\mathbb{R}^{4}$ which admits a pointwise $\mathrm{SO}(2)$-, $S_{3^{-}}$or $\mathbb{Z}_{3}$-symmetry. Let $p \in M$. Then there exists an orthonormal frame $\{T, V, W\}$ defined in a neighborhood of the point $p$ such that $K$ is given by:

$$
\begin{array}{lll}
K(T, T)=-2 a_{4} T, & K(T, V)=a_{4} V, & K(T, W)=a_{4} W, \\
K(V, V)=-a_{4} T+a_{6} V, & K(V, W)=-a_{6} W, & K(W, W)=-a_{4} T-a_{6} V,
\end{array}
$$

where $a_{4}>0$ and $a_{6}=0$ in case of $\mathrm{SO}(2)$-symmetry, $a_{4}=0$ and $a_{6}>0$ in case of $S_{3}$-symmetry, and $a_{4}>0$ and $a_{6}>0$ in case of $\mathbb{Z}_{3}$-symmetry.

Proof. First we want to show that at every point the vector $\mathbf{t}$ is uniquely defined (up to sign) and differentiable. We introduce a symmetric operator $\hat{A}$ by:

$$
\widehat{\operatorname{Ric}}(Y, Z)=h(\hat{A} Y, Z) \text {. }
$$

Clearly $\hat{A}$ is a differentiable operator on $M$. Since $2\left(H-3 a_{4}^{2}\right) \neq 2\left(H-a_{4}^{2}+a_{6}^{2}\right)$, the operator has two distinct eigenvalues. A standard result then implies that the eigen distributions are differentiable. We take $T$ a local unit vector field spanning the 1-dimensional eigen distribution, and local orthonormal vector fields $\tilde{V}$ and $\tilde{W}$ spanning the second eigen distribution. If $a_{6}=0$, we can take $V=\tilde{V}$ and $W=\tilde{W}$.

As $T$ is (up to sign) uniquely determined, for $a_{6} \neq 0$ there exist differentiable functions $a_{4}, c_{6}$ and $c_{7}, c_{6}^{2}+c_{7}^{2} \neq 0$, such that

$$
\begin{array}{ll}
K(T, T)=-2 a_{4} T, & K(\tilde{V}, \tilde{V})=-a_{4} T+c_{6} \tilde{V}+c_{7} \tilde{W}, \\
K(T, \tilde{V})=a_{4} \tilde{V}, & K(\tilde{V}, \tilde{W})=c_{7} \tilde{V}-c_{6} \tilde{W}, \\
K(T, \tilde{W})=a_{4} \tilde{W}, & K(\tilde{W}, \tilde{W})=-a_{4} T-c_{6} \tilde{V}-c_{7} \tilde{W} .
\end{array}
$$

As we have shown in [13], in the proof of Theorem 2 (Case 2), we can always rotate $\tilde{V}$ and $\tilde{W}$ such that we obtain the desired frame.

Remark 1. It actually follows from the proof of the previous lemma that the vector field $T$ is (up to sign) invariantly defined on $M$, and therefore the function $a_{4}$, too. Since the Pick invariant (8) $J=\frac{1}{3}\left(-5 a_{4}^{2}+2 a_{6}^{2}\right)$, the function $a_{6}$ also is invariantly defined on the affine hypersphere $M^{3}$.

\section{Gauss and Codazzi for pointwise $\mathrm{SO}(2)-, S_{3^{-}}$or $\mathbb{Z}_{3}$-symmetry}

In this section we always will work with the local frame constructed in the previous lemma. We denote the coefficients of the Levi-Civita connection with respect to this frame by:

$$
\begin{array}{lll}
\widehat{\nabla}_{T} T=a_{12} V+a_{13} W, & \widehat{\nabla}_{T} V=a_{12} T-b_{13} W, & \widehat{\nabla}_{T} W=a_{13} T+b_{13} V, \\
\widehat{\nabla}_{V} T=a_{22} V+a_{23} W, & \widehat{\nabla}_{V} V=a_{22} T-b_{23} W, & \widehat{\nabla}_{V} W=a_{23} T+b_{23} V, \\
\widehat{\nabla}_{W} T=a_{32} V+a_{33} W, & \widehat{\nabla}_{W} V=a_{32} T-b_{33} W, & \widehat{\nabla}_{W} W=a_{33} T+b_{33} V .
\end{array}
$$

We will evaluate first the Codazzi and then the Gauss equations ((12) and (13)) to obtain more information.

Lemma 3. Let $M^{3}$ be an affine hypersphere in $\mathbb{R}^{4}$ which admits a pointwise $\mathrm{SO}(2)$-, $S_{3^{-}}$or $\mathbb{Z}_{3}$-symmetry and $\{T, V, W\}$ the corresponding ONB. If the symmetry group is 
$\mathbf{S O}(\mathbf{2})$, then $0=a_{12}=a_{13}=a_{23}=a_{32}, a_{33}=a_{22}$ and

$$
T\left(a_{4}\right)=-4 a_{22} a_{4}, 0=V\left(a_{4}\right)=W\left(a_{4}\right),
$$

$\mathbf{S}_{\mathbf{3}}$, then $0=a_{12}=a_{13}, a_{23}=-3 b_{13}=-a_{32}, a_{33}=a_{22}$ and

$$
T\left(a_{6}\right)=-a_{22} a_{6}, V\left(a_{6}\right)=3 b_{33} a_{6}, W\left(a_{6}\right)=-3 b_{23} a_{6},
$$

$\mathbb{Z}_{\mathbf{3}}$ and $\mathbf{a}_{\mathbf{6}}^{\mathbf{2}} \neq \mathbf{4} \mathbf{a}_{\mathbf{4}}^{\mathbf{2}}$, then $0=a_{12}=a_{13}=a_{23}=a_{32}, a_{33}=a_{22}, b_{13}=0$,

$T\left(a_{4}\right)=-4 a_{22} a_{4}, 0=V\left(a_{4}\right)=W\left(a_{4}\right)$, and

$T\left(a_{6}\right)=-a_{22} a_{6}, V\left(a_{6}\right)=3 b_{33} a_{6}, W\left(a_{6}\right)=-3 b_{23} a_{6}$,

$\mathbb{Z}_{\mathbf{3}}$ and $\mathbf{a}_{\mathbf{6}}=\mathbf{2 \mathbf { a } _ { \mathbf { 4 } }}$, then $a_{12}=2 a_{22}=-2 a_{33}=-b_{33}$,

$a_{13}=-2 a_{23}=-2 a_{32}=b_{23}, b_{13}=0$, and

$T\left(a_{4}\right)=0, V\left(a_{4}\right)=-4 a_{22} a_{4}, W\left(a_{4}\right)=4 a_{23} a_{4}$.

Proof. An evaluation of the Codazzi equations (12) with the help of the CAS Mathematica leads to the following equations (they relate to eq1-eq6 and eq8-eq9 in the Mathematica notebook):

$$
\begin{aligned}
& V\left(a_{4}\right)=-2 a_{12} a_{4}, \quad T\left(a_{4}\right)=-4 a_{22} a_{4}+a_{12} a_{6}, \quad 0=4 a_{23} a_{4}+a_{13} a_{6}, \\
& W\left(a_{4}\right)=-2 a_{13} a_{4}, \quad 0=4 a_{32} a_{4}+a_{13} a_{6}, \quad T\left(a_{4}\right)=-4 a_{33} a_{4}-a_{12} a_{6}, \\
& T\left(a_{6}\right)-V\left(a_{4}\right)=3 a_{12} a_{4}-a_{22} a_{6}, \quad 0=a_{13} a_{4}+\left(a_{23}+3 b_{13}\right) a_{6}, \\
& W\left(a_{4}\right)=\left(a_{23}+a_{32}\right) a_{6}, \quad W\left(a_{6}\right)=\left(-a_{23}+3 a_{32}\right) a_{4}-b_{23} a_{6}, \\
& V\left(a_{6}\right)=\left(-a_{22}+a_{33}\right) a_{4}+3 b_{33} a_{6}, \\
& T\left(a_{6}\right)=-a_{12} a_{4}-a_{33} a_{6}, \quad W\left(a_{4}\right)=-3 a_{13} a_{4}+\left(-a_{32}+3 b_{13}\right) a_{6}, \\
& V\left(a_{4}\right)=\left(-a_{22}+a_{33}\right) a_{6}, \quad W\left(a_{6}\right)=\left(3 a_{23}-a_{32}\right) a_{4}-3 b_{23} a_{6}, \\
& 0=\left(a_{23}-a_{32}\right) a_{4}, \\
& W\left(a_{4}\right)=-a_{13} a_{4}+\left(a_{32}-3 b_{13}\right) a_{6} .
\end{aligned}
$$

From the first equation of (15) (we will use the notation (15).1) and (17).1 resp. (14).3 and (15).2 we get:

$$
0=2 a_{13} a_{4}+\left(a_{23}+a_{32}\right) a_{6}, \quad 0=2\left(a_{23}+a_{32}\right) a_{4}+a_{13} a_{6} .
$$

From (19).1) and (14).1 resp. (14).2 and (15).3 we get:

$$
0=-2 a_{12} a_{4}+2\left(a_{22}-a_{33}\right) a_{6}, \quad 0=2\left(-a_{22}+a_{33}\right) a_{4}+a_{12} a_{6} .
$$

We consider first the case, that $\mathbf{a}_{\mathbf{6}}^{\mathbf{2}} \neq \mathbf{4} \mathbf{a}_{\mathbf{4}}^{\mathbf{2}}$. Then we obtain from the foregoing equations that $a_{13}=0, a_{32}=-a_{23}, a_{12}=0$ and $a_{33}=a_{22}$. Furthermore it follows from (14). 1 that $V\left(a_{4}\right)=0$, from (14).2 that $T\left(a_{4}\right)=-4 a_{22} a_{4}$ and from (14).3 that $a_{23} a_{4}=0$. Equation (15).1 becomes $W\left(a_{4}\right)=0$, equation (16).2 $T\left(a_{6}\right)=-a_{22} a_{6}$ and (16).3 $\left(a_{23}+3 b_{13}\right) a_{6}=0$. Finally equation (17). 2 resp. 3 gives $W\left(a_{6}\right)=-3 b_{23} a_{6}$ and $V\left(a_{6}\right)=3 b_{33} a_{6}$.

In case of $S O(2)$-symmetry $\left(a_{4}>0\right.$ and $\left.a_{6}=0\right)$ it follows that $a_{23}=0$ and thus the statement of the theorem.

In case of $S_{3}$-symmetry $\left(a_{4}=0\right.$ and $\left.a_{6}>0\right)$ it follows that $a_{23}=-3 b_{13}$ and thus the statement of the theorem.

In case of $\mathbb{Z}_{3}$-symmetry $\left(a_{4}>0\right.$ and $\left.a_{6}>0\right)$ it follows that $a_{23}=0$ and $b_{13}=0$ and thus the statement of the theorem.

In case that $a_{6}= \pm 2 a_{4}(\neq 0)$, we can choose $V, W$ such that $\mathbf{a}_{\mathbf{6}}=\mathbf{2} \mathbf{a}_{\mathbf{4}}$. Now equations (20), (14).3 and (16).3 lead to $a_{23}=a_{32}, a_{13}=-2 a_{23}$ and $b_{13}=0$. A combination of (14).2 and (15).3 gives $a_{12}=\left(a_{22}-a_{33}\right)$, and then by equations (16).2, (14).1 and (14).2 that $a_{33}=-a_{22}$. Thus $T\left(a_{4}\right)=0$ by (14).2, $V\left(a_{4}\right)=-4 a_{22} a_{4}$ by (14).1 and $W\left(a_{4}\right)=4 a_{22} a_{4}$ by (15).1. Finally (17). 2 and (15). 1 resp. (17). 3 and (14). 1 imply that $b_{23}=-a_{23}$ resp. $b_{33}=-a_{22}$. 
An evaluation of the Gauss equations (13) with the help of the CAS Mathematica leads to the following:

Lemma 4. Let $M^{3}$ be an affine hypersphere in $\mathbb{R}^{4}$ which admits a pointwise $\mathrm{SO}(2)$-, $S_{3}$ - or $\mathbb{Z}_{3}$-symmetry and $\{T, V, W\}$ the corresponding ONB. Then

$$
\begin{aligned}
& T\left(a_{22}\right)=-a_{22}^{2}+a_{23}^{2}+H-3 a_{4}^{2}, \\
& T\left(a_{23}\right)=-2 a_{22} a_{23}, \\
& W\left(a_{22}\right)+V\left(a_{23}\right)=0, \\
& W\left(a_{23}\right)-V\left(a_{22}\right)=0, \\
& V\left(b_{13}\right)-T\left(b_{23}\right)=a_{22} b_{23}+\left(a_{23}+b_{13}\right) b_{33}, \\
& T\left(b_{33}\right)-W\left(b_{13}\right)=\left(a_{23}+b_{13}\right) b_{23}-a_{22} b_{33}, \\
& V\left(b_{33}\right)-W\left(b_{23}\right)=-a_{22}^{2}-a_{23}^{2}+2 a_{23} b_{13}+b_{23}^{2}+b_{33}^{2}+H+a_{4}^{2}+2 a_{6}^{2} .
\end{aligned}
$$

If the symmetry group is $\mathbb{Z}_{3}$, then $a_{6}^{2} \neq 4 a_{4}^{2}$.

Proof. The equations relate to eq11-eq13 and eq16 in the Mathematica notebook. If $a_{6}^{2}=$ $4 a_{4}^{2}(\neq 0)$, then we obtain by equations eq11.1 and eq12.3 resp. eq15.3 and eq12.3 that $2 V\left(a_{22}\right)=$ $-4 a_{22}^{2}-H+3 a_{4}^{2}$ resp. $2 W\left(a_{23}\right)=4 a_{23}^{2}+H-3 a_{4}^{2}$, thus $V\left(a_{22}\right)-W\left(a_{23}\right)=-2 a_{22}^{2}-2 a_{23}^{2}-H+3 a_{4}^{2}$. This gives a contradiction to eq13.3, namely $V\left(a_{22}\right)-W\left(a_{23}\right)=-2 a_{22}^{2}-2 a_{23}^{2}-H-9 a_{4}^{2}$.

\section{$5 \quad$ Pointwise $\mathbb{Z}_{3^{-}}$or $\mathrm{SO}(2)$-symmetry}

The following methods only work in the case of $\mathbb{Z}_{3^{-}}$or $\mathrm{SO}(2)$-symmetry, therefore the case of $S_{3^{-}}$ symmetry will be considered elsewhere. As the vector field $T$ is globally defined, we can define the distributions $L_{1}=\operatorname{span}\{T\}$ and $L_{2}=\operatorname{span}\{V, W\}$. In the following we will investigate these distributions. For the terminology we refer to [9].

Lemma 5. The distribution $L_{1}$ is autoparallel (totally geodesic) with respect to $\widehat{\nabla}$.

Proof. From $\widehat{\nabla}_{T} T=a_{12} V+a_{13} W=0$ (cf. Lemmas 3 and 4 ) the claim follows immediately.

Lemma 6. The distribution $L_{2}$ is spherical with mean curvature normal $U_{2}=a_{22} T$.

Proof. For $U_{2}=a_{22} T \in L_{1}=L_{2}^{\perp}$ we have $h\left(\widehat{\nabla}_{E_{a}} E_{b}, T\right)=h\left(E_{a}, E_{b}\right) h\left(U_{2}, T\right)$ for $E_{a}, E_{b} \in$ $\{V, W\}$, and $h\left(\widehat{\nabla}_{E_{a}} U_{2}, T\right)=h\left(E_{a}\left(a_{22}\right) T+a_{22} \widehat{\nabla}_{E_{a}} T, T\right)=0$ (cf. Lemma 3 and (26), (27)).

Remark 2. $a_{22}$ is independent of the choice of $\mathrm{ONB}\{V, W\}$. It therefore is a globally defined function on $M^{3}$.

We introduce a coordinate function $t$ by $\frac{\partial}{\partial t}:=T$. Using the previous lemma, according to [12], we get:

Lemma 7. $\left(M^{3}, h\right)$ admits a warped product structure $M^{3}=I \times{ }_{e^{f}} N^{2}$ with $f: I \rightarrow \mathbb{R}$ satisfying

$$
\frac{\partial f}{\partial t}=a_{22}
$$

Proof. Proposition 3 in [12] gives the warped product structure with warping function $\lambda_{2}$ : $I \rightarrow \mathbb{R}$. If we introduce $f=\ln \lambda_{2}$, following the proof we see that $a_{22} T=U_{2}=-\operatorname{grad}\left(\ln \lambda_{2}\right)=$ $-\operatorname{grad} f$.

Lemma 8. The curvature of $N^{2}$ is ${ }^{N} K\left(N^{2}\right)=e^{2 f}\left(H+2 a_{6}^{2}+a_{4}^{2}-a_{22}^{2}\right)$. 
Proof. From Proposition 2 in [12] we get the following relation between the curvature tensor $\hat{R}$ of the warped product $M^{3}$ and the curvature tensor $\tilde{R}$ of the usual product of pseudo-Riemannian manifolds $(X, Y, Z \in \mathcal{X}(M)$ resp. their appropriate projections):

$$
\begin{aligned}
\hat{R}(X, Y) Z= & \tilde{R}(X, Y) Z+h(Y, Z)\left(\widehat{\nabla}_{X} U_{2}-h\left(X, U_{2}\right) U_{2}\right)-h\left(\widehat{\nabla}_{X} U_{2}-h\left(X, U_{2}\right) U_{2}, Z\right) Y \\
& -h(X, Z)\left(\widehat{\nabla}_{Y} U_{2}-h\left(Y, U_{2}\right) U_{2}\right)+h\left(\widehat{\nabla}_{Y} U_{2}-h\left(Y, U_{2}\right) U_{2}, Z\right) X \\
& +h\left(U_{2}, U_{2}\right)(h(Y, Z) X-h(X, Z) Y) .
\end{aligned}
$$

Now $\tilde{R}(X, Y) Z={ }^{N} \hat{R}(X, Y) Z$ for all $X, Y, Z \in T N^{2}$ and otherwise zero (cf. [11, page 89], Corollary 58) and $K\left(N^{2}\right)=K(V, W)=\frac{h(-\hat{R}(V, W) V, W)}{h(V, V) h(W, W)-h(V, W)^{2}}$ (cf. [11, page 77], the curvature tensor has the opposite sign). Since $h(X, Y)=e^{2 f N} h(X, Y)$ for $X, Y \in T N^{2}$, it follows that

$$
{ }^{N} K\left(N^{2}\right)=e^{2 f} h\left(-{ }^{N} \hat{R}(V, W) V, W\right) .
$$

Finally we obtain by the Gauss equation (13) the last ingredient for the computation: $\hat{R}(V, W) V$ $=-\left(H+2 a_{6}^{2}+a_{4}^{2}\right) W$ (cf. the Mathematica notebook).

Summarized we have obtained the following structure equations (cf. (1), (2) and (3)), where $a_{6}=0$ in case of $\mathrm{SO}(2)$-symmetry resp. $b_{13}=0$ in case of $\mathbb{Z}_{3}$-symmetry:

$$
\begin{aligned}
& D_{T} T=-2 a_{4} T-\xi, \\
& D_{T} V=+a_{4} V-b_{13} W, \\
& D_{T} W=+b_{13} V+a_{4} W, \\
& D_{V} T=+\left(a_{22}+a_{4}\right) V, \\
& D_{W} T=+\left(a_{22}+a_{4}\right) W, \\
& D_{V} V=+a_{6} V-b_{23} W+\left(a_{22}-a_{4}\right) T+\xi, \\
& D_{V} W=+b_{23} V-a_{6} W, \\
& D_{W} V=-\left(b_{33}+a_{6}\right) W, \\
& D_{W} W=+\left(b_{33}-a_{6}\right) V+\left(a_{22}-a_{4}\right) T+\xi, \\
& D_{X} \xi=-H X .
\end{aligned}
$$

The Codazzi and Gauss equations ((12) and (13)) have the form (cf. Lemmas 3 and 4):

$$
\begin{aligned}
& T\left(a_{4}\right)=-4 a_{22} a_{4}, \quad 0=V\left(a_{4}\right)=W\left(a_{4}\right), \\
& T\left(a_{6}\right)=-a_{22} a_{6}, \quad V\left(a_{6}\right)=3 b_{33} a_{6}, \quad W\left(a_{6}\right)=-3 b_{23} a_{6}, \\
& T\left(a_{22}\right)=-a_{22}^{2}+H-3 a_{4}^{2}, \quad V\left(a_{22}\right)=0, \quad W\left(a_{22}\right)=0, \\
& V\left(b_{13}\right)-T\left(b_{23}\right)=a_{22} b_{23}+b_{13} b_{33}, \\
& T\left(b_{33}\right)-W\left(b_{13}\right)=b_{13} b_{23}-a_{22} b_{33}, \\
& V\left(b_{33}\right)-W\left(b_{23}\right)=-a_{22}^{2}+b_{23}^{2}+b_{33}^{2}+H+a_{4}^{2}+2 a_{6}^{2},
\end{aligned}
$$

where $a_{6}=0$ in case of $\mathrm{SO}(2)$-symmetry resp. $b_{13}=0$ in case of $\mathbb{Z}_{3}$-symmetry.

Our first goal is to find out how $N^{2}$ is immersed in $\mathbb{R}^{4}$, i.e. to find an immersion independent of $t$. A look at the structure equations (32)-(41) suggests to start with a linear combination of $T$ and $\xi$.

We will solve the problem in two steps. First we look for a vector field $X$ with $D_{T} X=\alpha X$ for some function $\alpha$ : We define $X:=A T+\xi$ for some function $A$ on $M^{3}$. Then $D_{T} X=\alpha X$ iff $\alpha=-A$ and $\frac{\partial}{\partial t} A=-A^{2}+2 a_{4} A+H$, and $A:=a_{22}-a_{4}$ solves the latter differential equation. 
Next we want to multiply $X$ with some function $\beta$ such that $D_{T}(\beta X)=0$ : We define a positive function $\beta$ on $\mathbb{R}$ as the solution of the differential equation:

$$
\frac{\partial}{\partial t} \beta=\left(a_{22}-a_{4}\right) \beta
$$

with initial condition $\beta\left(t_{0}\right)>0$. Then $D_{T}(\beta X)=0$ and by (35), (41) and (36) we get (since $\beta$, $a_{22}$ and $a_{4}$ only depend on $t$ ):

$$
\begin{aligned}
& D_{T}\left(\beta\left(\left(a_{22}-a_{4}\right) T+\xi\right)\right)=0, \\
& D_{V}\left(\beta\left(\left(a_{22}-a_{4}\right) T+\xi\right)\right)=\beta\left(a_{22}^{2}-a_{4}^{2}-H\right) V, \\
& D_{W}\left(\beta\left(\left(a_{22}-a_{4}\right) T+\xi\right)\right)=\beta\left(a_{22}^{2}-a_{4}^{2}-H\right) W .
\end{aligned}
$$

To obtain an immersion we need that $\nu:=a_{22}^{2}-a_{4}^{2}-H$ vanishes nowhere, but we only get:

Lemma 9. The function $\nu=a_{22}^{2}-a_{4}^{2}-H$ is globally defined, $\frac{\partial}{\partial t}\left(e^{2 f} \nu\right)=0$ and $\nu$ vanishes identically or nowhere on $\mathbb{R}$.

Proof. Since $0=\frac{\partial}{\partial t}^{N} K\left(N^{2}\right)=\frac{\partial}{\partial t}\left(e^{2 f}\left(2 a_{6}^{2}-\nu\right)\right.$ ) (Lemma 8) and $\frac{\partial}{\partial t}\left(e^{2 f} 2 a_{6}^{2}\right)=0$ (cf. (43) and (31)), we get that $\frac{\partial}{\partial t}\left(e^{2 f} \nu\right)=0$. Thus $\frac{\partial}{\partial t} \nu=-2\left(\frac{\partial}{\partial t} f\right) \nu=-2 a_{22} \nu$.

\subsection{The first case: $\nu \neq 0$ on $M^{3}$}

We may, by translating $f$, i.e. by replacing $N^{2}$ with a homothetic copy of itself, assume that $e^{2 f} \nu=\varepsilon_{1}$, where $\varepsilon_{1}= \pm 1$.

Lemma 10. $\Phi:=\beta\left(\left(a_{22}-a_{4}\right) T+\xi\right): M^{3} \rightarrow \mathbb{R}^{4}$ induces a proper affine sphere structure, say $\tilde{\phi}$, mapping $N^{2}$ into a 3-dimensional linear subspace of $\mathbb{R}^{4}$. $\tilde{\phi}$ is part of a quadric iff $a_{6}=0$.

Proof. By (50) and (51) we have $\Phi_{*}\left(E_{a}\right)=\beta \nu E_{a}$ for $E_{a} \in\{V, W\}$. A further differentiation, using (37) ( $\beta$ and $\nu$ only depend on $t$ ), gives:

$$
\begin{aligned}
D_{V} \Phi_{*}(V) & =\beta \nu D_{V} V=\beta \nu\left(\left(a_{22}-a_{4}\right) T+a_{6} V-b_{23} W+\xi\right) \\
& =a_{6} \Phi_{*}(V)-b_{23} \Phi_{*}(W)+\nu \Phi=a_{6} \Phi_{*}(V)-b_{23} \Phi_{*}(W)+\varepsilon_{1} e^{-2 f} \Phi .
\end{aligned}
$$

Similarly, we obtain the other derivatives, using (38)-(40), thus:

$$
\begin{aligned}
& D_{V} \Phi_{*}(V)=a_{6} \Phi_{*}(V)-b_{23} \Phi_{*}(W)+e^{-2 f} \varepsilon_{1} \Phi, \\
& D_{V} \Phi_{*}(W)=b_{23} \Phi_{*}(V)-a_{6} \Phi_{*}(W), \\
& D_{W} \Phi_{*}(V)=-\left(b_{33}+a_{6}\right) \Phi_{*}(W), \\
& D_{W} \Phi_{*}(W)=\left(b_{33}-a_{6}\right) \Phi_{*}(V)+e^{-2 f} \varepsilon_{1} \Phi, \\
& D_{E_{a}} \Phi=\beta e^{-2 f} \varepsilon_{1} E_{a} .
\end{aligned}
$$

The foliation at $f=f_{0}$ gives an immersion of $N^{2}$ to $M^{3}$, say $\pi_{f_{0}}$. Therefore, we can define an immersion of $N^{2}$ to $\mathbb{R}^{4}$ by $\tilde{\phi}:=\Phi \circ \pi_{f_{0}}$, whose structure equations are exactly the equations above when $f=f_{0}$. Hence, we know that $\tilde{\phi}$ maps $N^{2}$ into $\operatorname{span}\left\{\Phi_{*}(V), \Phi_{*}(W), \Phi\right\}$, an affine hyperplane of $\mathbb{R}^{4}$ and $\frac{\partial}{\partial t} \Phi=0$ implies $\Phi(t, v, w)=\tilde{\phi}(v, w)$.

We can read off the coefficients of the difference tensor $K^{\tilde{\phi}}$ of $\tilde{\phi}$ (cf. (1) and (3)): $K^{\tilde{\phi}}(\tilde{V}, \tilde{V})=$ $a_{6} \tilde{V}, K^{\tilde{\phi}}(\tilde{V}, \tilde{W})=-a_{6} \tilde{W}, K^{\tilde{\phi}}(\tilde{W}, \tilde{W})=-a_{6} \tilde{V}$, and see that trace $\left(K^{\tilde{\phi}}\right)_{X}$ vanishes. The affine metric introduced by this immersion corresponds with the metric on $N^{2}$. Thus $\varepsilon_{1} \tilde{\phi}$ is the affine normal of $\tilde{\phi}$ and $\tilde{\phi}$ is a proper affine sphere with mean curvature $\varepsilon_{1}$. Finally the vanishing of the difference tensor characterizes quadrics. 
Our next goal is to find another linear combination of $T$ and $\xi$, this time only depending on $t$. (Then we can express $T$ in terms of $\phi$ and some function of $t$.)

Lemma 11. Define $\delta:=H T+\left(a_{22}+a_{4}\right) \xi$. Then there exist a constant vector $C \in \mathbb{R}^{4}$ and a function $a(t)$ such that

$$
\delta(t)=a(t) C
$$

Proof. Using (35) resp. (36) and (41) we obtain that $D_{V} \delta=0=D_{W} \delta$. Hence $\delta$ depends only on the variable $t$. Moreover, we get by (32), (44), (42) and (41) that

$$
\begin{aligned}
\frac{\partial}{\partial t} \delta & =D_{T}\left(H T+\left(a_{22}+a_{4}\right) \xi\right) \\
& =H\left(-2 a_{4} T-\xi\right)+\left(-a_{22}^{2}+H-3 a_{4}^{2}-4 a_{22} a_{4}\right) \xi-\left(a_{22}+a_{4}\right) H T \\
& =-\left(3 a_{4}+a_{22}\right)\left(H T+\left(a_{22}+a_{4}\right) \xi\right)=-\left(3 a_{4}+a_{22}\right) \delta .
\end{aligned}
$$

This implies that there exists a constant vector $C$ in $\mathbb{R}^{4}$ and a function $a(t)$ such that $\delta(t)=$ $a(t) C$.

Notice that for an improper affine hypersphere $(H=0) \xi$ is constant and parallel to $C$. Combining $\tilde{\phi}$ and $\delta$ we obtain for $T$ (cf. Lemmas 10 and 11) that

$$
T(t, v, w)=-\frac{a}{\nu} C+\frac{1}{\beta \nu}\left(a_{22}+a_{4}\right) \tilde{\phi}(v, w) .
$$

In the following we will use for the partial derivatives the abbreviation $\varphi_{x}:=\frac{\partial}{\partial x} \varphi, x=t, v, w$.

\section{Lemma 12.}

$$
\varphi_{t}=-\frac{a}{\nu} C+\frac{\partial}{\partial t}\left(\frac{1}{\beta \nu}\right) \tilde{\phi}, \quad \varphi_{v}=\frac{1}{\beta \nu} \tilde{\phi}_{v}, \quad \varphi_{w}=\frac{1}{\beta \nu} \tilde{\phi}_{w}
$$

Proof. As by (48) and Lemma $9 \frac{\partial}{\partial t} \frac{1}{\beta \nu}=\frac{1}{\beta \nu}\left(a_{22}+a_{4}\right)$, we obtain the equation for $\varphi_{t}=T$ by (57). The other equations follow from (50) and (51).

It follows by the uniqueness theorem of first order differential equations and applying a translation that we can write

$$
\varphi(t, v, w)=\tilde{a}(t) C+\frac{1}{\beta \nu}(t) \tilde{\phi}(v, w)
$$

for a suitable function $\tilde{a}$ depending only on the variable $t$. Since $C$ is transversal to the image of $\tilde{\phi}$ (cf. Lemmas 10 and 11, $\nu \neq \equiv 0$ ), we obtain that after applying an equiaffine transformation we can write: $\varphi(t, v, w)=\left(\gamma_{1}(t), \gamma_{2}(t) \phi(v, w)\right)$, in which $\tilde{\phi}(v, w)=(0, \phi(v, w))$. Thus we have proven the following:

Theorem 1. Let $M^{3}$ be an indefinite affine hypersphere of $\mathbb{R}^{4}$ which admits a pointwise $\mathbb{Z}_{3}$ - or $S O(2)$-symmetry. Let $a_{22}^{2}-a_{4}^{2} \neq H$ for some $p \in M^{3}$. Then $M^{3}$ is affine equivalent to

$$
\varphi: I \times N^{2} \rightarrow \mathbb{R}^{4}:(t, v, w) \mapsto\left(\gamma_{1}(t), \gamma_{2}(t) \phi(v, w)\right),
$$

where $\phi: N^{2} \rightarrow \mathbb{R}^{3}$ is a (positive definite) elliptic or hyperbolic affine sphere and $\gamma: I \rightarrow \mathbb{R}^{2}$ is a curve. Moreover, if $M^{3}$ admits a pointwise $S O(2)$-symmetry then $N^{2}$ is either an ellipsoid or a two-sheeted hyperboloid. 
We want to investigate the conditions imposed on the curve $\gamma$. For this we compute the derivatives of $\varphi$ :

$$
\begin{array}{lll}
\varphi_{t}=\left(\gamma_{1}^{\prime}, \gamma_{2}^{\prime} \phi\right), & \varphi_{v}=\left(0, \gamma_{2} \phi_{v}\right), & \varphi_{w}=\left(0, \gamma_{2} \phi_{w}\right), \\
\varphi_{t t}=\left(\gamma_{1}^{\prime \prime}, \gamma_{2}^{\prime \prime} \phi\right), & \varphi_{t v}=\left(0, \gamma_{2}^{\prime} \phi_{v}\right), & \varphi_{t w}=\left(0, \gamma_{2}^{\prime} \phi_{w}\right), \\
\varphi_{v v}=\left(0, \gamma_{2} \phi_{v v}\right), & \varphi_{v w}=\left(0, \gamma_{2} \phi_{v w}\right), & \varphi_{w w}=\left(0, \gamma_{2}^{\prime} \phi_{w w}\right) .
\end{array}
$$

Furthermore we have to distinguish if $M^{3}$ is proper $(H= \pm 1)$ or improper $(H=0)$.

First we consider the case that $M^{3}$ is proper, i.e. $\xi=-H \varphi$. An easy computation shows that the condition that $\xi$ is a transversal vector field, namely $0 \neq \operatorname{det}\left(\varphi_{t}, \varphi_{v}, \varphi_{w}, \xi\right)=-\gamma_{2}^{2}\left(\gamma_{1} \gamma_{2}^{\prime}-\right.$ $\left.\gamma_{1}^{\prime} \gamma_{2}\right) \operatorname{det}\left(\phi_{v}, \phi_{w}, \phi\right)$, is equivalent to $\gamma_{2} \neq 0$ and $\gamma_{1} \gamma_{2}^{\prime}-\gamma_{1}^{\prime} \gamma_{2} \neq 0$. To check the condition that $\xi$ is the Blaschke normal (cf. (4)), we need to compute the Blaschke metric $h$, using (1), (58), (52)-(55) and the notation $r, s \in\{v, w\}$ and $g$ for the Blaschke metric of $\phi$ :

$$
\begin{aligned}
& \varphi_{t t}=\cdots \varphi_{t}+\frac{\gamma_{1}^{\prime} \gamma_{2}^{\prime \prime}-\gamma_{1}^{\prime \prime} \gamma_{2}^{\prime}}{H\left(\gamma_{1} \gamma_{2}^{\prime}-\gamma_{1}^{\prime} \gamma_{2}\right)} \xi, \quad \varphi_{t r}=\operatorname{tang}, \\
& \varphi_{r s}=\operatorname{tang}-\frac{\gamma_{1}^{\prime} \gamma_{2}}{H\left(\gamma_{1} \gamma_{2}^{\prime}-\gamma_{1}^{\prime} \gamma_{2}\right)} \varepsilon_{1} g\left(\frac{\partial}{\partial r}, \frac{\partial}{\partial s}\right) \xi .
\end{aligned}
$$

We obtain that

$$
\operatorname{det} h=h_{t t}\left(h_{v v} h_{w w}-h_{v w}^{2}\right)=\frac{\gamma_{1}^{\prime} \gamma_{2}^{\prime \prime}-\gamma_{1}^{\prime \prime} \gamma_{2}^{\prime}}{H^{3}\left(\gamma_{1} \gamma_{2}^{\prime}-\gamma_{1}^{\prime} \gamma_{2}\right)^{3}}\left(\gamma_{1}^{\prime}\right)^{2} \gamma_{2}^{2} \operatorname{det} g
$$

Thus

$$
\gamma_{2}^{4}\left(\gamma_{1} \gamma_{2}^{\prime}-\gamma_{1}^{\prime} \gamma_{2}\right)^{2} \operatorname{det}\left(\phi_{v}, \phi_{w}, \phi\right)^{2}=\left|\frac{\gamma_{1}^{\prime} \gamma_{2}^{\prime \prime}-\gamma_{1}^{\prime \prime} \gamma_{2}^{\prime}}{\left(\gamma_{1} \gamma_{2}^{\prime}-\gamma_{1}^{\prime} \gamma_{2}\right)^{3}}\left(\gamma_{1}^{\prime}\right)^{2} \gamma_{2}^{2} \operatorname{det} g\right|
$$

is equivalent to (4). Since $\phi$ is a definite proper affine sphere with normal $-\varepsilon_{1} \phi$, we can again use (4) to obtain

$$
\xi=-H \varphi \Longleftrightarrow \gamma_{2}^{2}\left|\gamma_{1} \gamma_{2}^{\prime}-\gamma_{1}^{\prime} \gamma_{2}\right|^{5}=\left|\gamma_{1}^{\prime} \gamma_{2}^{\prime \prime}-\gamma_{1}^{\prime \prime} \gamma_{2}^{\prime}\right|\left(\gamma_{1}^{\prime}\right)^{2} \neq 0
$$

From the computations above ( $g$ is positive definite) also it follows that $\varphi$ is indefinite iff either

$$
\begin{aligned}
& H \operatorname{sign}\left(\gamma_{1} \gamma_{2}^{\prime}-\gamma_{1}^{\prime} \gamma_{2}\right)=\operatorname{sign}\left(\gamma_{1}^{\prime} \gamma_{2}^{\prime \prime}-\gamma_{1}^{\prime \prime} \gamma_{2}^{\prime}\right)=\operatorname{sign}\left(\gamma_{1}^{\prime} \gamma_{2} \varepsilon_{1}\right) \quad \text { or } \\
& -H \operatorname{sign}\left(\gamma_{1} \gamma_{2}^{\prime}-\gamma_{1}^{\prime} \gamma_{2}\right)=\operatorname{sign}\left(\gamma_{1}^{\prime} \gamma_{2}^{\prime \prime}-\gamma_{1}^{\prime \prime} \gamma_{2}^{\prime}\right)=\operatorname{sign}\left(\gamma_{1}^{\prime} \gamma_{2} \varepsilon_{1}\right) .
\end{aligned}
$$

Next we consider the case that $M^{3}$ is improper, i.e. $\xi$ is constant. By Lemma $11 \xi$ is parallel to $C$ and thus transversal to $\phi$. Hence we can apply an affine transformation to obtain $\xi=(1,0,0,0)$. An easy computation shows that the condition that $\xi$ is a transversal vector field, namely $0 \neq \operatorname{det}\left(\varphi_{t}, \varphi_{v}, \varphi_{w}, \xi\right)=-\gamma_{2}^{2} \gamma_{2}^{\prime} \operatorname{det}\left(\phi_{v}, \phi_{w}, \phi\right)$, is equivalent to $\gamma_{2} \neq 0$ and $\gamma_{2}^{\prime} \neq 0$. To check the condition that $\xi$ is the Blaschke normal (cf. (4)) we need to compute the Blaschke metric $h$, using (1), (58), (52)-(55) and the notation $r, s \in\{v, w\}$ and $g$ for the Blaschke metric of $\phi$ :

$$
\varphi_{t t}=\cdots \varphi_{t}-\frac{\gamma_{1}^{\prime} \gamma_{2}^{\prime \prime}-\gamma_{1}^{\prime \prime} \gamma_{2}^{\prime}}{\gamma_{2}^{\prime}} \xi, \quad \varphi_{t r}=\operatorname{tang}, \quad \varphi_{r s}=\operatorname{tang}+\frac{\gamma_{1}^{\prime} \gamma_{2}}{\gamma_{2}^{\prime}} \varepsilon_{1} g\left(\frac{\partial}{\partial r}, \frac{\partial}{\partial s}\right) \xi
$$

We obtain that

$$
\operatorname{det} h=h_{t t}\left(h_{v v} h_{w w}-h_{v w}^{2}\right)=-\frac{\gamma_{1}^{\prime} \gamma_{2}^{\prime \prime}-\gamma_{1}^{\prime \prime} \gamma_{2}^{\prime}}{\left(\gamma_{2}^{\prime}\right)^{3}}\left(\gamma_{1}^{\prime}\right)^{2} \gamma_{2}^{2} \operatorname{det} g .
$$


Thus (4) is equivalent to

$$
\gamma_{2}^{4}\left(\gamma_{2}^{\prime}\right)^{2} \operatorname{det}\left(\phi_{v}, \phi_{w}, \phi\right)^{2}=\left|\frac{\gamma_{1}^{\prime} \gamma_{2}^{\prime \prime}-\gamma_{1}^{\prime \prime} \gamma_{2}^{\prime}}{\left(\gamma_{2}^{\prime}\right)^{3}}\left(\gamma_{1}^{\prime}\right)^{2} \gamma_{2}^{2} \operatorname{det} g\right|
$$

Since $\phi$ is a definite proper affine sphere with normal $-\varepsilon_{1} \phi$, we can again use (4) to obtain

$$
\xi=(1,0,0,0) \Longleftrightarrow \gamma_{2}^{2}\left|\gamma_{2}^{\prime}\right|^{5}=\left|\gamma_{1}^{\prime} \gamma_{2}^{\prime \prime}-\gamma_{1}^{\prime \prime} \gamma_{2}^{\prime}\right|\left(\gamma_{1}^{\prime}\right)^{2} \neq 0
$$

From the computations above also it follows that $\varphi$ is indefinite iff either

$$
\begin{aligned}
& -\operatorname{sign}\left(\gamma_{2}^{\prime}\right)=\operatorname{sign}\left(\gamma_{1}^{\prime} \gamma_{2}^{\prime \prime}-\gamma_{1}^{\prime \prime} \gamma_{2}^{\prime}\right)=\operatorname{sign}\left(\gamma_{1}^{\prime} \gamma_{2} \varepsilon_{1}\right) \quad \text { or } \\
& \operatorname{sign}\left(\gamma_{2}^{\prime}\right)=\operatorname{sign}\left(\gamma_{1}^{\prime} \gamma_{2}^{\prime \prime}-\gamma_{1}^{\prime \prime} \gamma_{2}^{\prime}\right)=\operatorname{sign}\left(\gamma_{1}^{\prime} \gamma_{2} \varepsilon_{1}\right)
\end{aligned}
$$

So we have seen under which conditions we can construct a 3-dimensional indefinite affine hypersphere out of an affine sphere:

Theorem 2. Let $\phi: N^{2} \rightarrow \mathbb{R}^{3}$ be a positive definite elliptic or hyperbolic affine sphere (with mean curvature $\left.\varepsilon_{1}= \pm 1\right)$, and let $\gamma=\left(\gamma_{1}, \gamma_{2}\right): I \rightarrow \mathbb{R}^{2}$ be a curve. Define $\varphi: I \times N^{2} \rightarrow \mathbb{R}^{4}$ by $\varphi(t, v, w)=\left(\gamma_{1}(t), \gamma_{2}(t) \phi(v, w)\right)$.

(i) If $\gamma$ satisfies $\gamma_{2}^{2}\left|\gamma_{1} \gamma_{2}^{\prime}-\gamma_{1}^{\prime} \gamma_{2}\right|^{5}=\operatorname{sign}\left(\gamma_{1}^{\prime} \gamma_{2} \varepsilon_{1}\right)\left(\gamma_{1}^{\prime} \gamma_{2}^{\prime \prime}-\gamma_{1}^{\prime \prime} \gamma_{2}^{\prime}\right)\left(\gamma_{1}^{\prime}\right)^{2} \neq 0$, then $\varphi$ defines a 3-dimensional indefinite proper affine hypersphere.

(ii) If $\gamma$ satisfies $\gamma_{2}^{2}\left|\gamma_{2}^{\prime}\right|^{5}=\operatorname{sign}\left(\gamma_{1}^{\prime} \gamma_{2} \varepsilon_{1}\right)\left(\gamma_{1}^{\prime} \gamma_{2}^{\prime \prime}-\gamma_{1}^{\prime \prime} \gamma_{2}^{\prime}\right)\left(\gamma_{1}^{\prime}\right)^{2} \neq 0$, then $\varphi$ defines a 3 -dimensional indefinite improper affine hypersphere.

Now we are ready to check the symmetries.

Theorem 3. Let $\phi: N^{2} \rightarrow \mathbb{R}^{3}$ be a positive definite elliptic or hyperbolic affine sphere (with mean curvature $\left.\varepsilon_{1}= \pm 1\right)$, and let $\gamma=\left(\gamma_{1}, \gamma_{2}\right): I \rightarrow \mathbb{R}^{2}$ be a curve such that $\varphi(t, v, w)=$ $\left(\gamma_{1}(t), \gamma_{2}(t) \phi(v, w)\right)$ defines a 3 -dimensional indefinite affine hypersphere. Then $\varphi\left(N^{2} \times I\right)$ admits a pointwise $\mathbb{Z}_{3}$ - or $S O(2)$-symmetry.

Proof. We already have shown that $\varphi$ defines a 3 -dimensional indefinite proper resp. improper affine hypersphere. To prove the symmetry we need to compute $K$. By assumption, $\phi$ is an affine sphere with Blaschke normal $\xi^{\phi}=-\varepsilon_{1} \phi$. For the structure equations (1) we use the notation $\phi_{r s}={ }^{\phi} \Gamma_{r s}^{u} \phi_{u}-g_{r s} \varepsilon_{1} \phi, r, s, u \in\{v, w\}$. Furthermore we introduce the notation $\alpha=\gamma_{1} \gamma_{2}^{\prime}-\gamma_{1}^{\prime} \gamma_{2}$. Note that $\alpha^{\prime}=\gamma_{1} \gamma_{2}^{\prime \prime}-\gamma_{1}^{\prime \prime} \gamma_{2}$. If $\varphi$ is proper, using (58), we get the structure equations (1) for $\varphi$ :

$$
\begin{aligned}
& \varphi_{t t}=\frac{\alpha^{\prime}}{\alpha} \varphi_{t}+\frac{\gamma_{1}^{\prime} \gamma_{2}^{\prime \prime}-\gamma_{1}^{\prime \prime} \gamma_{2}^{\prime}}{H \alpha} \xi, \quad \varphi_{t r}=\frac{\gamma_{2}^{\prime}}{\gamma_{2}} \varphi_{r}, \\
& \varphi_{r s}={ }^{\phi} \Gamma_{r s}^{u} \varphi_{u}-g_{r s} \varepsilon_{1} \frac{\gamma_{1} \gamma_{2}}{\alpha} \varphi_{t}-g_{r s} \varepsilon_{1} \frac{\gamma_{1}^{\prime} \gamma_{2}}{H \alpha} \xi .
\end{aligned}
$$

We compute $K$ using (6) and obtain:

$$
\begin{aligned}
& \left(\nabla_{\varphi_{t}} h\right)\left(\varphi_{r}, \varphi_{s}\right)=\left(\left(\frac{\gamma_{1} \gamma_{2}}{\alpha}\right)^{\prime} \frac{\alpha}{\gamma_{1} \gamma_{2}}-2 \frac{\gamma_{2}^{\prime}}{\gamma_{2}}\right) h\left(\varphi_{r}, \varphi_{s}\right), \\
& \left(\nabla_{\varphi_{r}} h\right)\left(\varphi_{t}, \varphi_{t}\right)=0,
\end{aligned}
$$

implying that $K_{\varphi_{t}}$ restricted to the space spanned by $\varphi_{v}$ and $\varphi_{w}$ is a multiple of the identity. Taking $T$ in direction of $\varphi_{t}$, we see that $\varphi_{v}$ and $\varphi_{w}$ are orthogonal to $T$. Thus we can construct an ONB $\{T, V, W\}$ with $V, W$ spanning $\operatorname{span}\left\{\varphi_{v}, \varphi_{w}\right\}$ such that $a_{1}=2 a_{4}, a_{2}=a_{3}=a_{5}=0$. By the considerations in $[13$, Section 4$]$ we see that $\varphi$ admits a pointwise $\mathbb{Z}_{3^{-}}$or $S O(2)$-symmetry. If $\varphi$ is improper, the proof runs completely analogous. 


\subsection{The second case: $\nu \equiv 0$ and $H \neq 0$ on $M^{3}$}

Next, we consider the case that $H=a_{22}^{2}-a_{4}^{2}$ and $H \neq 0$ on $M^{3}$. It follows that $a_{22} \neq \pm a_{4}$ on $M^{3}$.

We already have seen that $M^{3}$ admits a warped product structure. The map $\Phi$ we have constructed in Lemma 10 will not define an immersion (cf. (50) and (51)). Anyhow, for a fixed point $t_{0}$, we get from (37)-(40), (50) and (51), using the notation $\tilde{\xi}=\left(a_{22}-a_{4}\right) T+\xi$ :

$$
\begin{aligned}
& D_{V} V=a_{6} V-b_{23} W+\tilde{\xi}, \quad D_{V} W=b_{23} V-a_{6} W \\
& D_{W} V=-\left(b_{33}+a_{6}\right) W, \quad D_{W} W=\left(b_{33}-a_{6}\right) V+\tilde{\xi}, \quad D_{E_{a}} \tilde{\xi}=0, \quad E_{a} \in\{V, W\} .
\end{aligned}
$$

Thus, if $v$ and $w$ are local coordinates which span the second distribution $L_{2}$, then we can interpret $\varphi\left(t_{0}, v, w\right)$ as a positive definite improper affine sphere in a 3-dimensional linear subspace.

Moreover, we see that this improper affine sphere is a paraboloid provided that $a_{6}\left(t_{0}, v, w\right)$ vanishes identically. From the differential equations (43) determining $a_{6}$, we see that this is the case exactly when $a_{6}$ vanishes identically, i.e. when $M^{3}$ admits a pointwise $S O(2)$-symmetry.

After applying a translation and a change of coordinates, we may assume that

$$
\varphi\left(t_{0}, v, w\right)=(v, w, f(v, w), 0)
$$

with affine normal $\tilde{\xi}\left(t_{0}, v, w\right)=(0,0,1,0)$. To obtain $T$ at $t_{0}$, we consider (35) and (36) and get that

$$
D_{E_{a}}\left(T-\left(a_{22}+a_{4}\right) \varphi\right)=0, \quad E_{a}, E_{b} \in\{V, W\} .
$$

Evaluating at $t=t_{0}$, this means that there exists a constant vector $C$, transversal to $\operatorname{span}\{V, W, \xi\}$, such that $T\left(t_{0}, v, w\right)=\left(a_{22}+a_{4}\right)\left(t_{0}\right) \varphi\left(t_{0}, v, w\right)+C$. Since $a_{22}+a_{4} \neq 0$ everywhere, we can write:

$$
T\left(t_{0}, v, w\right)=\alpha_{1}\left(v, w, f(v, w), \alpha_{2}\right),
$$

where $\alpha_{1}, \alpha_{2} \neq 0$ and we applied an equiaffine transformation so that $C=\left(0,0,0, \alpha_{1} \alpha_{2}\right)$. To obtain information about $D_{T} T$ we have that $D_{T} T=-2 a_{4} T-\xi$ (cf. (32)) and $\xi=\tilde{\xi}-\left(a_{22}-a_{4}\right) T$ by the definition of $\tilde{\xi}$. Also we know that $\tilde{\xi}\left(t_{0}, v, w\right)=(0,0,1,0)$ and by $(49)-(51)$ that $D_{X}(\beta \tilde{\xi})=$ $0, X \in \mathcal{X}(M)$. Taking suitable initial conditions for the function $\beta\left(\beta\left(t_{0}\right)=1\right)$, we get that $\beta \tilde{\xi}=(0,0,1,0)$ and finally the following vector valued differential equation:

$$
D_{T} T=\left(a_{22}-3 a_{4}\right) T-\frac{1}{\beta}(0,0,1,0) .
$$

Solving this differential equation, taking into account the initial conditions (59) at $t=t_{0}$, we get that there exist functions $\delta_{1}$ and $\delta_{2}$ depending only on $t$ such that

$$
T(t, u, v)=\left(\delta_{1}(t) v, \delta_{1}(t) w, \delta_{1}(t)\left(f(v, w)+\delta_{2}(t)\right), \alpha_{2} \delta_{1}(t)\right)
$$

where $\delta_{1}\left(t_{0}\right)=\alpha_{1}, \delta_{2}\left(t_{0}\right)=0, \delta_{1}^{\prime}(t)=\left(a_{22}-3 a_{4}\right) \delta_{1}(t)$ and $\delta_{2}^{\prime}(t)=\delta_{1}^{-1}(t) \beta^{-1}(t)$. As $T(t, v, w)=$ $\frac{\partial \varphi}{\partial t}(t, v, w)$ and $\varphi\left(t_{0}, v, w\right)=(v, w, f(v, w), 0)$ it follows by integration that

$$
\varphi(t, v, w)=\left(\gamma_{1}(t) v, \gamma_{1}(t) w, \gamma_{1}(t) f(v, w)+\gamma_{2}(t), \alpha_{2}\left(\gamma_{1}(t)-1\right)\right)
$$

where $\gamma_{1}^{\prime}(t)=\delta_{1}(t), \gamma_{1}\left(t_{0}\right)=1, \gamma_{2}\left(t_{0}\right)=0$ and $\gamma_{2}^{\prime}(t)=\delta_{1}(t) \delta_{2}(t)$. After applying an affine transformation we have shown: 
Theorem 4. Let $M^{3}$ be an indefinite proper affine hypersphere of $\mathbb{R}^{4}$ which admits a pointwise $\mathbb{Z}_{3}$ - or $S O(2)$-symmetry. Let $H=a_{22}^{2}-a_{4}^{2}(\neq 0)$ on $M^{3}$. Then $M^{3}$ is affine equivalent with

$$
\varphi: I \times N^{2} \rightarrow \mathbb{R}^{4}:(t, v, w) \mapsto\left(\gamma_{1}(t) v, \gamma_{1}(t) w, \gamma_{1}(t) f(v, w)+\gamma_{2}(t), \gamma_{1}(t)\right),
$$

where $\psi: N^{2} \rightarrow \mathbb{R}^{3}:(v, w) \mapsto(v, w, f(v, w))$ is a positive definite improper affine sphere with affine normal $(0,0,1)$ and $\gamma: I \rightarrow \mathbb{R}^{2}$ is a curve. Moreover, if $M^{3}$ admits a pointwise $S O(2)$-symmetry then $N^{2}$ is an elliptic paraboloid.

We want to investigate the conditions imposed on the curve $\gamma$. For this we compute the derivatives of $\varphi$ :

$$
\begin{aligned}
& \varphi_{t}=\left(\gamma_{1}^{\prime} v, \gamma_{1}^{\prime} w, \gamma_{1}^{\prime} f(v, w)+\gamma_{2}^{\prime}, \gamma_{1}^{\prime}\right), \\
& \varphi_{v}=\left(\gamma_{1}, 0, \gamma_{1} f_{v}, 0\right), \quad \varphi_{w}=\left(0, \gamma_{1}, \gamma_{1} f_{w}, 0\right), \\
& \varphi_{t t}=\left(\gamma_{1}^{\prime \prime} v, \gamma_{1}^{\prime \prime} w, \gamma_{1}^{\prime \prime} f(v, w)+\gamma_{2}^{\prime \prime}, \gamma_{1}^{\prime \prime}\right), \\
& \varphi_{t v}=\frac{\gamma_{1}^{\prime}}{\gamma_{1}} \varphi_{v}, \quad \varphi_{t w}=\frac{\gamma_{1}^{\prime}}{\gamma_{1}} \varphi_{w}, \\
& \varphi_{v v}=\left(0,0, f_{v v} \gamma_{1}, 0\right), \quad \varphi_{v w}=\left(0,0, \gamma_{1} f_{v w}, 0\right), \quad \varphi_{w w}=\left(0,0, \gamma_{1} f_{w w}, 0\right) .
\end{aligned}
$$

$M^{3}$ is a proper hypersphere, i.e. $\xi=-H \varphi$. An easy computation shows that the condition that $\xi$ is a transversal vector field, namely $0 \neq \operatorname{det}\left(\varphi_{t}, \varphi_{v}, \varphi_{w}, \xi\right)=-H \gamma_{1}^{2}\left(\gamma_{1} \gamma_{2}^{\prime}-\gamma_{1}^{\prime} \gamma_{2}\right)$, is equivalent to $\gamma_{1} \neq 0$ and $\gamma_{1} \gamma_{2}^{\prime}-\gamma_{1}^{\prime} \gamma_{2} \neq 0$. Since $(0,0,1,0)=\frac{\gamma_{1}}{\gamma_{1} \gamma_{2}^{\prime}-\gamma_{1}^{\prime} \gamma_{2}} \varphi_{t}-\frac{\gamma_{1}^{\prime}}{\gamma_{1} \gamma_{2}^{\prime}-\gamma_{1}^{\prime} \gamma_{2}} \varphi$, we have the following structure equations:

$$
\begin{aligned}
& \varphi_{t t}=\left(\frac{\gamma_{1}^{\prime \prime}}{\gamma_{1}^{\prime}}+\frac{\gamma_{1}^{\prime} \gamma_{2}^{\prime \prime}-\gamma_{1}^{\prime \prime} \gamma_{2}^{\prime}}{\gamma_{1}^{\prime}} \frac{\gamma_{1}}{\gamma_{1} \gamma_{2}^{\prime}-\gamma_{1}^{\prime} \gamma_{2}}\right) \varphi_{t}+\frac{\gamma_{1}^{\prime} \gamma_{2}^{\prime \prime}-\gamma_{1}^{\prime \prime} \gamma_{2}^{\prime}}{\gamma_{1} \gamma_{2}^{\prime}-\gamma_{1}^{\prime} \gamma_{2}} \frac{1}{H} \xi \\
& \varphi_{t r}=\frac{\gamma_{1}^{\prime}}{\gamma_{1}} \varphi_{r}, \quad \varphi_{r s}=\frac{\gamma_{1}^{2}}{\gamma_{1} \gamma_{2}^{\prime}-\gamma_{1}^{\prime} \gamma_{2}} f_{r s} \varphi_{t}+\frac{\gamma_{1} \gamma_{1}^{\prime}}{\gamma_{1} \gamma_{2}^{\prime}-\gamma_{1}^{\prime} \gamma_{2}} f_{r s} \frac{1}{H} \xi .
\end{aligned}
$$

We obtain:

$$
\operatorname{det} h=h_{t t}\left(h_{v v} h_{w w}-h_{v w}^{2}\right)=\frac{\gamma_{1}^{\prime} \gamma_{2}^{\prime \prime}-\gamma_{1}^{\prime \prime} \gamma_{2}^{\prime}}{H^{3}\left(\gamma_{1} \gamma_{2}^{\prime}-\gamma_{1}^{\prime} \gamma_{2}\right)^{3}} \gamma_{1}^{2}\left(\gamma_{1}^{\prime}\right)^{2}\left(f_{v v} f_{w w}-f_{v w}^{2}\right)
$$

Since $\psi$ is a positive definite improper affine sphere with affine normal $(0,0,1)$, we get by $(4)$ that $f_{v v} f_{w w}-f_{v w}^{2}=1$. Now (4) (for $\xi$ ) is equivalent to

$$
\gamma_{1}^{4}\left(\gamma_{1} \gamma_{2}^{\prime}-\gamma_{1}^{\prime} \gamma_{2}\right)^{2}=\left|\frac{\gamma_{1}^{\prime} \gamma_{2}^{\prime \prime}-\gamma_{1}^{\prime \prime} \gamma_{2}^{\prime}}{\left(\gamma_{1} \gamma_{2}^{\prime}-\gamma_{1}^{\prime} \gamma_{2}\right)^{3}}\right| \gamma_{1}^{2}\left(\gamma_{1}^{\prime}\right)^{2}
$$

It follows that

$$
\xi=-H \varphi \Longleftrightarrow \gamma_{1}^{2}\left|\gamma_{1} \gamma_{2}^{\prime}-\gamma_{1}^{\prime} \gamma_{2}\right|^{5}=\left|\gamma_{1}^{\prime} \gamma_{2}^{\prime \prime}-\gamma_{1}^{\prime \prime} \gamma_{2}^{\prime}\right|\left(\gamma_{1}^{\prime}\right)^{2} \neq 0
$$

From the computations above also it follows that $\varphi$ is indefinite iff either

$$
\begin{aligned}
& \operatorname{sign}\left(\gamma_{1}^{\prime} \gamma_{2}^{\prime \prime}-\gamma_{1}^{\prime \prime} \gamma_{2}^{\prime}\right)=\operatorname{sign}\left(H\left(\gamma_{1} \gamma_{2}^{\prime}-\gamma_{1}^{\prime} \gamma_{2}\right)\right)=-\operatorname{sign}\left(\gamma_{1} \gamma_{1}^{\prime}\right) \quad \text { or } \\
& \operatorname{sign}\left(\gamma_{1}^{\prime} \gamma_{2}^{\prime \prime}-\gamma_{1}^{\prime \prime} \gamma_{2}^{\prime}\right)=-\operatorname{sign}\left(H\left(\gamma_{1} \gamma_{2}^{\prime}-\gamma_{1}^{\prime} \gamma_{2}\right)\right)=-\operatorname{sign}\left(\gamma_{1} \gamma_{1}^{\prime}\right) .
\end{aligned}
$$

So we have seen under which conditions we can construct a 3-dimensional indefinite affine hypersphere out of an affine sphere:

Theorem 5. Let $\psi: N^{2} \rightarrow \mathbb{R}^{3}:(v, w) \mapsto(v, w, f(v, w))$ be a positive definite improper affine sphere with affine normal $(0,0,1)$, and let $\gamma: I \rightarrow \mathbb{R}^{2}$ be a curve. Define $\varphi: I \times N^{2} \rightarrow \mathbb{R}^{4}$ by $\varphi(t, v, w)=\left(\gamma_{1}(t) v, \gamma_{1}(t) w, \gamma_{1}(t) f(v, w)+\gamma_{2}(t), \gamma_{1}(t)\right)$. If $\gamma=\left(\gamma_{1}, \gamma_{2}\right)$ satisfies $\gamma_{1}^{2}\left|\gamma_{1} \gamma_{2}^{\prime}-\gamma_{1}^{\prime} \gamma_{2}\right|^{5}=$ $-\operatorname{sign}\left(\gamma_{1} \gamma_{1}^{\prime}\right)\left(\gamma_{1}^{\prime} \gamma_{2}^{\prime \prime}-\gamma_{1}^{\prime \prime} \gamma_{2}^{\prime}\right)\left(\gamma_{1}^{\prime}\right)^{2} \neq 0$, then $\varphi$ defines a 3-dimensional indefinite proper affine hypersphere. 
Now we are ready to check the symmetries.

Theorem 6. Let $\psi: N^{2} \rightarrow \mathbb{R}^{3}:(v, w) \mapsto(v, w, f(v, w))$ be a positive definite improper affine sphere with affine normal $(0,0,1)$, and let $\gamma: I \rightarrow \mathbb{R}^{2}$ be a curve such that $\varphi(t, v, w)=$ $\left(\gamma_{1}(t) v, \gamma_{1}(t) w, \gamma_{1}(t) f(v, w)+\gamma_{2}(t), \gamma_{1}(t)\right)$ defines a 3-dimensional indefinite proper affine hypersphere. Then $\varphi\left(N^{2} \times I\right)$ admits a pointwise $\mathbb{Z}_{3}$ - or $S O(2)$-symmetry.

Proof. We already have shown that $\varphi$ defines a 3-dimensional indefinite proper affine hypersphere with affine normal $\xi=-H \varphi$. To prove the symmetry we need to compute $K$. We get the induced connection and the affine metric from the structure equations (61). We compute $K$ using (6) and obtain:

$$
\begin{aligned}
\left(\nabla_{\varphi_{t}} h\right)\left(\varphi_{r}, \varphi_{s}\right) & =\left(\frac{\partial}{\partial t} \ln \left(\frac{\gamma_{1} \gamma_{1}^{\prime}}{\gamma_{1} \gamma_{2}^{\prime}-\gamma_{1}^{\prime} \gamma_{2}}\right)-2 \frac{\gamma_{1}^{\prime}}{\gamma_{1}}\right) h\left(\varphi_{r}, \varphi_{s}\right), \\
\left(\nabla_{\varphi_{r}} h\right)\left(\varphi_{t}, \varphi_{t}\right) & =0
\end{aligned}
$$

implying that $K_{\varphi_{t}}$ restricted to the space spanned by $\varphi_{v}$ and $\varphi_{w}$ is a multiple of the identity. Taking $T$ in direction of $\varphi_{t}$, we see that $\varphi_{v}$ and $\varphi_{w}$ are orthogonal to $T$. Thus we can construct an ONB $\{T, V, W\}$ with $V, W$ spanning $\operatorname{span}\left\{\varphi_{v}, \varphi_{w}\right\}$ such that $a_{1}=2 a_{4}, a_{2}=a_{3}=a_{5}=0$. By the considerations in $[13$, Section 4$]$ we see that $\varphi$ admits a pointwise $\mathbb{Z}_{3}$ - or $S O(2)$-symmetry.

\subsection{The third case: $\nu \equiv 0$ and $H=0$ on $M^{3}$}

The final cases now are that $\nu \equiv 0$ and $H=0$ on the whole of $M^{3}$ and hence $a_{22}= \pm a_{4}$.

First we consider the case that $a_{22}=a_{4}=: a>0$. Again we use that $M^{3}$ admits a warped product structure and we fix a parameter $t_{0}$. At the point $t_{0}$, we have by (37)-(41):

$$
\begin{aligned}
& D_{V} V=+a_{6} V-b_{23} W+\xi, \\
& D_{V} W=+b_{23} V-a_{6} W, \\
& D_{W} V=-\left(b_{33}+a_{6}\right) W, \\
& D_{W} W=+\left(b_{33}-a_{6}\right) V+\xi, \\
& D_{X} \xi=0 .
\end{aligned}
$$

Thus, if $v$ and $w$ are local coordinates which span the second distribution $L_{2}$, then we can interpret $\varphi\left(t_{0}, v, w\right)$ as a positive definite improper affine sphere in a 3 -dimensional linear subspace.

Moreover, we see that this improper affine sphere is a paraboloid provided that $a_{6}\left(t_{0}, v, w\right)$ vanishes identically. From the differential equations (43) determining $a_{6}$, we see that this is the case exactly when $a_{6}$ vanishes identically, i.e. when $M^{3}$ admits a pointwise $S O(2)$-symmetry.

After applying an affine transformation and a change of coordinates, we may assume that

$$
\varphi\left(t_{0}, v, w\right)=(v, w, f(v, w), 0),
$$

with affine normal $\xi\left(t_{0}, v, w\right)=(0,0,1,0)$, actually

$$
\xi(t, v, w)=(0,0,1,0)
$$

( $\xi$ is constant on $M^{3}$ by assumption). Furthermore we obtain by (35) and (36), that $D_{U} T=2 a U$ for all $U \in L_{2}$. We define $\delta:=T-2 a \varphi$, which is transversal to $\operatorname{span}\{V, W, \xi\}$. Since $a$ is independent of $v$ and $w$ (cf. (42)), $D_{U} \delta=0$, and we can assume that

$$
T\left(t_{0}, v, w\right)-2 a\left(t_{0}\right) \varphi\left(t_{0}, v, w\right)=(0,0,0,1) .
$$


We can integrate (42) $\left(T(a)=-4 a^{2}\right)$ and we take $a=\frac{1}{4 t}, t>0$. Thus (32) becomes $D_{T} T=$ $-\frac{1}{2 t} T-\xi$ and we obtain the following linear second order ordinary differential equation:

$$
\frac{\partial^{2}}{\partial t^{2}} \varphi+\frac{1}{2 t} \frac{\partial}{\partial t} \varphi=-\xi
$$

The general solution is $\varphi(t, v, w)=-\frac{t^{2}}{3} \xi+2 \sqrt{t} A(v, w)+B(v, w)$. The initial conditions (62) and (63) imply that $A(v, w)=\left(\frac{v}{2 \sqrt{t_{0}}}, \frac{w}{2 \sqrt{t_{0}}}, \frac{f(v, w)}{2 \sqrt{t_{0}}}+\frac{2}{3} t_{0}^{3 / 2}, \sqrt{t_{0}}\right)$ and $B(v, w)=\left(0,0,-t_{0}^{2},-2 t_{0}\right)$. Obviously we can translate $B$ to zero. Furthermore we can translate the affine sphere and apply an affine transformation to obtain $A(v, w)=\frac{1}{2 \sqrt{t_{0}}}(v, w, f(v, w), 1)$. After a change of coordinates we get:

$$
\varphi(t, v, w)=\left(t v, t w, t f(v, w)-c t^{4}, t\right), \quad c, t>0 .
$$

Next we consider the case that $-a_{22}=a_{4}=: a>0$. Again we use that $M^{3}$ admits a warped product structure and we fix a parameter $t_{0}$. A look at (37)-(41) suggests to define $\tilde{\xi}=-2 a T+\xi$, then we get at the point $t_{0}$ :

$$
\begin{aligned}
& D_{V} V=+a_{6} V-b_{23} W+\tilde{\xi}, \\
& D_{V} W=+b_{23} V-a_{6} W, \\
& D_{W} V=-\left(b_{33}+a_{6}\right) W, \\
& D_{W} W=+\left(b_{33}-a_{6}\right) V+\tilde{\xi}, \\
& D_{U} \tilde{\xi}=0 .
\end{aligned}
$$

Thus, if $v$ and $w$ are local coordinates which span the second distribution $L_{2}$, then we can interpret $\varphi\left(t_{0}, v, w\right)$ as a positive definite improper affine sphere in a 3 -dimensional linear subspace.

Moreover, we see that this improper affine sphere is a paraboloid provided that $a_{6}\left(t_{0}, v, w\right)$ vanishes identically. From the differential equations (43) determining $a_{6}$, we see that this is the case exactly when $a_{6}$ vanishes identically, i.e. when $M^{3}$ admits a pointwise $S O(2)$-symmetry.

After applying an affine transformation and a change of coordinates, we may assume that

$$
\varphi\left(t_{0}, v, w\right)=(v, w, f(v, w), 0),
$$

with affine normal

$$
\tilde{\xi}\left(t_{0}, v, w\right)=(0,0,1,0) .
$$

We have considered $\tilde{\xi}$ before. We can solve (48) $\left(\frac{\partial}{\partial t} \beta=-2 a \beta\right)$ explicitly by $\beta=c \frac{1}{\sqrt{a}}$ (cf. (42)) and get by $(49)-(51)$ that $D_{X}\left(\frac{1}{\sqrt{a}} \tilde{\xi}\right)=0$. Thus $\frac{1}{\sqrt{a}}(-2 a T+\xi)=: C$ for a constant vector $C$, i.e. $T=-\frac{1}{2 a}(\sqrt{a} C-\xi)$. Notice that by $(41) \xi$ is a constant vector, too. We can choose $a=\frac{1}{4|t|}$, $t<0$ (cf. (42)), and we obtain the ordinary differential equation:

$$
\frac{\partial}{\partial t} \varphi=-\sqrt{|t|} C-2 t \xi, \quad t<0 .
$$

The solution (after a translation) with respect to the initial condition $(66)$ is $\varphi(t, v, w)=\frac{2}{3}|t|^{\frac{3}{2}} C-$ $t^{2} \xi+(v, w, f(v, w), 0)$. Notice that $C$ is a multiple of $\tilde{\xi}$ and hence by (67) a constant multiple of $(0,0,1,0)$. Furthermore $\xi$ is transversal to the space spanned by $\varphi\left(t_{0}, v, w\right)$. So we get after an affine transformation and a change of coordinates:

$$
\varphi(t, v, w)=\left(v, w, f(v, w)+c t^{3}, t^{4}\right), \quad c, t>0
$$

Combining both results (65) and (69) we have: 
Theorem 7. Let $M^{3}$ be an indefinite improper affine hypersphere of $\mathbb{R}^{4}$ which admits a pointwise $\mathbb{Z}_{3}$ - or $S O(2)$-symmetry. Let $a_{22}^{2}=a_{4}^{2}$ on $M^{3}$. Then $M^{3}$ is affine equivalent with either

$$
\begin{array}{llr}
\varphi: I \times N^{2} \rightarrow \mathbb{R}^{4}:(t, v, w) & \mapsto\left(t v, t w, t f(v, w)-c t^{4}, t\right), \quad\left(a_{22}=a_{4}\right) \quad \text { or } \\
\varphi: I \times N^{2} \rightarrow \mathbb{R}^{4}:(t, v, w) \mapsto\left(v, w, f(v, w)+c t^{3}, t^{4}\right), \quad\left(-a_{22}=a_{4}\right), &
\end{array}
$$

where $\psi: N^{2} \rightarrow \mathbb{R}^{3}:(v, w) \mapsto(v, w, f(v, w))$ is a positive definite improper affine sphere with affine normal $(0,0,1)$ and $c, t \in \mathbb{R}^{+}$. Moreover, if $M^{3}$ admits a pointwise $S O(2)$-symmetry then $N^{2}$ is an elliptic paraboloid.

The computations for the converse statement can be done completely analogous to the previous cases, they even are simpler (the curve is given parametrized).

Theorem 8. Let $\psi: N^{2} \rightarrow \mathbb{R}^{3}:(v, w) \mapsto(v, w, f(v, w))$ be a positive definite improper affine sphere with affine normal $(0,0,1)$. Define $\varphi(t, v, w)=\left(t v, t w, t f(v, w)-c t^{4}, t\right)$ or $\varphi(t, v, w)=$ $\left(v, w, f(v, w)+c t^{3}, t^{4}\right)$, where $c, t \in \mathbb{R}^{+}$. Then $\varphi$ defines a 3-dimensional indefinite improper affine hypersphere, which admits a pointwise $\mathbb{Z}_{3}$ - or $S O(2)$-symmetry.

\section{Pointwise SO $(1,1)$-symmetry}

Let $M^{3}$ be a hypersphere admitting a $\mathrm{SO}(1,1)$-symmetry. We only state the classification results. The proofs are done quite similar, using a lightvector-frame instead of an orthonormal one, and will appear elsewhere. We denote a lightvector-frame by $\{E, V, F\}$, where $E$ and $F$ are lightvectors and $V$ is spacelike (cf. [13]).

Lemma 13. Let $M^{3}$ be an affine hypersphere in $\mathbb{R}^{4}$ which admits a pointwise $\mathrm{SO}(1,1)$-symmetry. Let $p \in M$. Then there exists a lightvector-frame $\{E, V, F\}$ defined in a neighborhood of the point $p$ and a positive function $b_{4}$ such that $K$ is given by:

$$
\begin{array}{lll}
K(V, V)=-2 b_{4} V, & K(V, E)=b_{4} E, & K(V, F)=b_{4} F, \\
K(E, E)=0, & K(E, F)=b_{4} V, & K(F, F)=0 .
\end{array}
$$

In the following we denote the coefficients of the Levi-Civita connection with respect to this frame by:

$$
\begin{array}{llll}
\widehat{\nabla}_{E} E=a_{11} E+b_{11} V, & \widehat{\nabla}_{E} V=a_{12} E-b_{11} F, & \widehat{\nabla}_{E} F=-a_{12} V-a_{11} F, \\
\widehat{\nabla}_{V} E=a_{21} E+b_{21} V, & \widehat{\nabla}_{V} V=a_{22} E-b_{21} F, & \widehat{\nabla}_{V} F=-a_{22} V-a_{21} F, \\
\widehat{\nabla}_{F} E=a_{31} E+b_{31} V, & \widehat{\nabla}_{F} V=a_{32} E-b_{31} F, & & \widehat{\nabla}_{F} F=-a_{32} V-a_{31} F .
\end{array}
$$

Similar as before, it turns out that the vector field $V$ is globally defined, and we can define the distributions $L_{1}=\operatorname{span}\{V\}$ and $L_{2}=\operatorname{span}\{E, F\}$. Again, $L_{1}$ is autoparallel with respect to $\widehat{\nabla}$, and $L_{2}$ is spherical with mean curvature normal $-a_{12} V$. We introduce a coordinate function $v$ by $\frac{\partial}{\partial v}:=V$.

Lemma 14. The function $\nu=b_{4}^{2}-a_{12}^{2}-H$ is globally defined, $\frac{\partial}{\partial v}\left(e^{2 f} \nu\right)=0$ and $\nu$ vanishes identically or nowhere on $\mathbb{R}$.

Again we have to distinguish three cases. 


\subsection{The first case: $\nu \neq 0$ on $M^{3}$}

Theorem 9. Let $M^{3}$ be an indefinite affine hypersphere of $\mathbb{R}^{4}$ which admits a pointwise $S O(1,1)$ symmetry. Let $b_{4}^{2}-a_{12}^{2} \neq H$ for some $p \in M^{3}$. Then $M^{3}$ is affine equivalent to

$$
\varphi: I \times N^{2} \rightarrow \mathbb{R}^{4}:(v, x, y) \mapsto\left(\gamma_{1}(v), \gamma_{2}(v) \phi(x, y)\right),
$$

where $\phi: N^{2} \rightarrow \mathbb{R}^{3}$ is a one-sheeted hyperboloid and $\gamma: I \rightarrow \mathbb{R}^{2}$ is a curve.

Theorem 10. Let $\phi: N^{2} \rightarrow \mathbb{R}^{3}$ be a one-sheeted hyperboloid and let $\gamma: I \rightarrow \mathbb{R}^{2}$ be a curve. Define $\varphi: I \times N^{2} \rightarrow \mathbb{R}^{4}$ by $\varphi(v, x, y)=\left(\gamma_{1}(v), \gamma_{2}(v) \phi(x, y)\right)$.

(i) If $\gamma=\left(\gamma_{1}, \gamma_{2}\right)$ satisfies $\gamma_{2}^{2}\left|\gamma_{1} \gamma_{2}^{\prime}-\gamma_{1}^{\prime} \gamma_{2}\right|^{5}=\left|\gamma_{1}^{\prime} \gamma_{2}^{\prime \prime}-\gamma_{1}^{\prime \prime} \gamma_{2}^{\prime}\right|\left(\gamma_{1}^{\prime}\right)^{2} \neq 0$, then $\varphi$ defines a 3 -dimensional indefinite proper affine hypersphere.

(ii) If $\gamma=\left(\gamma_{1}, \gamma_{2}\right)$ satisfies $\gamma_{2}^{2}\left|\gamma_{2}^{\prime}\right|^{5}=\left|\gamma_{1}^{\prime} \gamma_{2}^{\prime \prime}-\gamma_{1}^{\prime \prime} \gamma_{2}^{\prime}\right|\left(\gamma_{1}^{\prime}\right)^{2} \neq 0$, then $\varphi$ defines a 3 -dimensional indefinite improper affine hypersphere.

Theorem 11. Let $\phi: N^{2} \rightarrow \mathbb{R}^{3}$ be a one-sheeted hyperboloid and let $\gamma: I \rightarrow \mathbb{R}^{2}$ be a curve, such that $\varphi(v, x, y)=\left(\gamma_{1}(v), \gamma_{2}(v) \phi(x, y)\right)$ defines a 3-dimensional indefinite affine hypersphere. Then $\varphi\left(I \times N^{2}\right)$ admits a pointwise $S O(1,1)$-symmetry.

\subsection{The second case: $\nu \equiv 0$ and $H \neq 0$ on $M^{3}$}

Theorem 12. Let $M^{3}$ be an indefinite proper affine hypersphere of $\mathbb{R}^{4}$ which admits a pointwise $S O(1,1)$-symmetry. Let $H=b_{4}^{2}-a_{12}^{2}(\neq 0)$ on $M^{3}$. Then $M^{3}$ is affine equivalent with

$$
\varphi: I \times N^{2} \rightarrow \mathbb{R}^{4}:(v, x, y) \mapsto\left(\gamma_{1}(v) x, \gamma_{1}(v) y, \gamma_{1}(v) f(x, y)+\gamma_{2}(v), \gamma_{1}(v)\right),
$$

where $\psi: N^{2} \rightarrow \mathbb{R}^{3}:(x, y) \mapsto(x, y, f(x, y))$ is a hyperbolic paraboloid with affine normal $(0,0,1)$ and $\gamma: I \rightarrow \mathbb{R}^{2}$ is a curve.

Theorem 13. Let $\psi: N^{2} \rightarrow \mathbb{R}^{3}$ be a hyperbolic paraboloid with affine normal $(0,0,1)$, and let $\gamma: I \rightarrow \mathbb{R}^{2}$ be a curve. Define $\varphi: I \times N^{2} \rightarrow \mathbb{R}^{4}$ by $\varphi(v, x, y)=\left(\gamma_{1}(v) x, \gamma_{1}(v) y, \gamma_{1}(v) f(x, y)+\right.$ $\left.\gamma_{2}(v), \gamma_{1}(v)\right)$. If $\gamma=\left(\gamma_{1}, \gamma_{2}\right)$ satisfies $\gamma_{1}^{2}\left|\gamma_{1} \gamma_{2}^{\prime}-\gamma_{1}^{\prime} \gamma_{2}\right|^{5}=\left|\gamma_{1}^{\prime} \gamma_{2}^{\prime \prime}-\gamma_{1}^{\prime \prime} \gamma_{2}^{\prime}\right|\left(\gamma_{1}^{\prime}\right)^{2} \neq 0$, then $\varphi$ defines a 3-dimensional indefinite proper affine hypersphere.

Theorem 14. Let $\psi: N^{2} \rightarrow \mathbb{R}^{3}$ be a hyperbolic paraboloid with affine normal $(0,0,1)$, and let $\gamma: I \rightarrow \mathbb{R}^{2}$ be a curve, such that $\varphi(v, x, y)=\left(\gamma_{1}(v) x, \gamma_{1}(v) y, \gamma_{1}(v) f(x, y)+\gamma_{2}(v), \gamma_{1}(v)\right)$ defines a 3-dimensional indefinite proper affine hypersphere. Then $\varphi\left(I \times N^{2}\right)$ admits a pointwise $S O(1,1)$-symmetry.

\subsection{The third case: $\nu \equiv 0$ and $H=0$ on $M^{3}$}

Theorem 15. Let $M^{3}$ be an indefinite improper affine hypersphere of $\mathbb{R}^{4}$ which admits a pointwise $S O(1,1)$-symmetry. Let $a_{12}^{2}=b_{4}^{2}$ on $M^{3}$. Then $M^{3}$ is affine equivalent with either

$$
\begin{array}{llll}
\varphi: I \times N^{2} \rightarrow \mathbb{R}^{4}:(v, x, y) \mapsto\left(v x, v y, v f(x, y)-c v^{4}, v\right), & \left(a_{12}=b_{4}\right) & \text { or } \\
\varphi: I \times N^{2} \rightarrow \mathbb{R}^{4}: & (v, x, y) \mapsto\left(x, y, f(x, y)+c v^{3}, v^{4}\right), & \left(-a_{12}=b_{4}\right), &
\end{array}
$$

where $\psi: N^{2} \rightarrow \mathbb{R}^{3}:(v, w) \mapsto(v, w, f(v, w))$ is a hyperbolic paraboloid with affine normal $(0,0,1)$ and $c, t \in \mathbb{R}^{+}$.

Theorem 16. Let $\psi: N^{2} \rightarrow \mathbb{R}^{3}:(v, w) \mapsto(v, w, f(v, w))$ be a hyperbolic paraboloid with affine normal $(0,0,1)$. Define $\varphi(t, v, w)=\left(t v, t w, t f(v, w)-c t^{4}, t\right)$ or $\varphi(t, v, w)=(v, w, f(v, w)+$ $\left.c t^{3}, t^{4}\right)$, where $t \in \mathbb{R}^{+}, c \neq 0$. Then $\varphi$ defines a 3 -dimensional indefinite improper affine hypersphere, which admits a pointwise $S O(1,1)$-symmetry. 


\section{A Computations for pointwise $\mathrm{SO}(2)-, S_{3^{-}}$or $\mathbb{Z}_{3^{-} \text {-Symmetry }}$}

$e[1]:=\{1,0,0\} ; e[2]:=\{0,1,0\} ; e[3]:=\{0,0,1\} ;$

$\mathrm{ONB}$ of $\mathrm{SO}(1,2): e[1]=\mathrm{T}, e[2]=\mathrm{V}, e[3]=\mathrm{W}$

\section{Affine metric}

$\mathrm{h}\left[\mathrm{y}_{-}, \mathrm{z}_{-}\right]:=-\mathrm{y}[[1]] \mathrm{z}[[1]]+\operatorname{Sum}[\mathrm{y}[[\mathrm{i}]] \mathrm{z}[[\mathrm{i}]],\{\mathrm{i}, 2,3\}] ;$

Difference tensor $(\mathrm{r}=\mathrm{a} 1, \mathrm{~s}=\mathrm{a} 4, \mathrm{u}=\mathrm{a} 6)$

$\mathrm{K}\left[\mathrm{y}_{-}, \mathrm{z}_{-}\right]:=\operatorname{Sum}[\mathrm{y}[[\mathrm{i}]] \mathrm{z}[[\mathrm{j}]] \mathrm{k}[\mathrm{i}, \mathrm{j}],\{\mathrm{i}, 1,3\},\{\mathrm{j}, 1,3\}] ;$

$\mathrm{k}[1,1]:=\{-\mathrm{r}, 0,0\} ; \mathrm{k}[1,2]:=\{0, \mathrm{~s}, 0\} ; \mathrm{k}[1,3]:=\{0,0, \mathrm{r}-\mathrm{s}\} ;$

$\mathrm{k}[2,1]:=\{0, \mathrm{~s}, 0\} ; \mathrm{k}[2,2]:=\{-\mathrm{s}, \mathrm{u}, 0\} ; \mathrm{k}[2,3]:=\{0,0,-\mathrm{u}\} ;$

$\mathrm{k}[3,1]:=\{0,0, \mathrm{r}-\mathrm{s}\} ; \mathrm{k}[3,2]:=\{0,0,-\mathrm{u}\} ; \mathrm{k}[3,3]:=\{-(\mathrm{r}-\mathrm{s}),-\mathrm{u}, 0\} ;$

\section{Ricci tensor, scalar curvature and Pick invariant}

$[\mathrm{Kx}, \mathrm{Ky}] \mathrm{z}$

$\mathrm{LK}\left[\mathrm{x}_{-}, \mathrm{y}_{-}, \mathrm{z}_{-}\right]:=K[x, K[y, z]]-K[y, K[x, z]] ;$

ListLK: $=\{\operatorname{LK}[e[1], e[2], e[1]], \operatorname{LK}[e[1], e[2], e[2]], \operatorname{LK}[e[1], e[2], e[3]]$,

$\operatorname{LK}[e[1], e[3], e[1]], \operatorname{LK}[e[1], e[3], e[2]], \operatorname{LK}[e[1], e[3], e[3]]$,

$\operatorname{LK}[e[2], e[3], e[1]], \operatorname{LK}[e[2], e[3], e[2]], \operatorname{LK}[e[2], e[3], e[3]]\} ;$

FullSimplify[ListLK]

Curvature tensor (of the Levi-Civita connection)

$R\left[\mathrm{x}_{-}, \mathrm{y}_{-}, \mathrm{z}_{-}\right]:=H(h[y, z] x-h[x, z] y)-K[x, K[y, z]]+K[y, K[x, z]] ;$

ListR: $=\{R[e[1], e[2], e[1]], R[e[1], e[2], e[2]], R[e[1], e[2], e[3]]$,

$R[e[1], e[3], e[1]], R[e[1], e[3], e[2]], R[e[1], e[3], e[3]]$,

$R[e[2], e[3], e[1]], R[e[2], e[3], e[2]], R[e[2], e[3], e[3]]\}$;

Simplify[ListR]

\section{Ricci tensor (of the Levi-Civita connection)}

$\operatorname{ric}\left[\mathrm{x}_{-}, \mathrm{y}_{-}\right]:=\operatorname{Simplify}[(-h[R[e[1], x, y], e[1]]+h[R[e[2], x, y], e[2]]+h[R[e[3], x, y], e[3]])] ;$

Listric: $=\{\operatorname{ric}[e[1], e[1]], \operatorname{ric}[e[1], e[2]], \operatorname{ric}[e[1], e[3]], \operatorname{ric}[e[2], e[2]], \operatorname{ric}[e[2], e[3]], \operatorname{ric}[e[3], e[3]]\} ;$

Simplify[Listric]

\section{Scalar curvature (of the Levi-Civita connection)}

$\mathrm{sc}:=1 / 6(-\operatorname{ric}[e[1], e[1]]+\operatorname{ric}[e[2], e[2]]+\operatorname{ric}[e[3], e[3]]) ;$

Simplify[sc]

\section{Pick invariant}

$P:=1 / 6\left(-(k[1,1][[1]])^{\wedge} 2+(k[2,2][[2]])^{\wedge} 2+(k[3,3][[3]])^{\wedge} 2+3\left((k[1,1][[2]])^{\wedge} 2+(k[1,1][[3]])^{\wedge} 2-\right.\right.$ $\left.\left.(k[2,2][[1]])^{\wedge} 2-(k[3,3][[1]])^{\wedge} 2+(k[2,2][[3]])^{\wedge} 2+(k[3,3][[2]])^{\wedge} 2\right)-6(k[1,2][[3]])^{\wedge} 2\right) ;$

Simplify $[P]$ 


\section{Lemma 1}

$r=2 s ;$ Simplify[Listric]

Simplify $[P]$

\section{Lemma 8}

$R[e[2], e[3], e[2]]$

\section{Lemma 3}

\section{Levi-Civita connection (ONB)}

$n[1,1]:=\{0, \mathrm{a} 12, \mathrm{a} 13\} ; n[1,2]:=\{\mathrm{a} 12,0,-\mathrm{b} 13\} ; n[1,3]:=\{\mathrm{a} 13, \mathrm{~b} 13,0\} ;$

$n[2,1]:=\{0, \mathrm{a} 22, \mathrm{a} 23\} ; n[2,2]:=\{\mathrm{a} 22,0,-\mathrm{b} 23\} ; n[2,3]:=\{\mathrm{a} 23, \mathrm{~b} 23,0\} ;$

$n[3,1]:=\{0, \mathrm{a} 32, \mathrm{a} 33\} ; n[3,2]:=\{\mathrm{a} 32,0,-\mathrm{b} 33\} ; n[3,3]:=\{\mathrm{a} 33, \mathrm{~b} 33,0\} ;$

$\mathrm{Na}\left[\mathrm{y}_{-}, \mathrm{z}_{-}\right]:=\operatorname{Sum}[y[[i]] z[[j]] n[i, j],\{i, 1,3\},\{j, 1,3\}]+\operatorname{Sum}[y[[1]] \operatorname{Dt}[z[[i]], \mathrm{f1}] e[i]$

$+y[[2]] \operatorname{Dt}[z[[i]], \mathrm{f} 2] e[i]+y[[3]] \mathrm{Dt}[z[[i]], \mathrm{f} 3] e[i],\{i, 1,3\}]$;

\section{Codazzi for $\mathrm{K}$ (affine hypersphere)}

codazzi $\left[\mathrm{x}_{-}, \mathrm{y}_{-}, \mathrm{z}_{-}\right]:=\mathrm{Na}[x, K[y, z]]-K[\mathrm{Na}[x, y], z]-K[y, \mathrm{Na}[x, z]]-\mathrm{Na}[y, K[x, z]]+K[\mathrm{Na}[y, x], z]+$ $K[x, \mathrm{Na}[y, z]]$

eq1:=Simplify [codazzi $[e[2], e[1], e[1]]] ;$ eq2:=Simplify[codazzi $[e[3], e[1], e[1]]] ;$

eq3:=Simplify[codazzi[ $[e[1], e[2], e[2]]] ;$ eq4:=Simplify[codazzi $[e[3], e[2], e[2]]] ;$

eq5:=Simplify[codazzi[ $[e[1], e[3], e[3]]] ;$ eq6:=Simplify[codazzi[ $[e[2], e[3], e[3]]] ;$

eq7:=Simplify [codazzi $[e[1], e[2], e[3]]] ;$ eq8:=Simplify[codazzi $[e[2], e[3], e[1]]] ;$

eq9:=Simplify [codazzi[ $e[3], e[1], e[2]]]$;

eq: $=\{$ eq1, eq2, eq3, eq4, eq5, eq6, eq7, eq8, eq9 $\} ;$ eq

\section{1. case: $\mathrm{u}^{\wedge} \mathbf{2} \neq 4 \mathrm{~s}^{\wedge} \mathbf{2}$}

\section{conclusions from eq1,2,4:}

Simplify[eq2[[1]] - 2eq4[[1]]]

Simplify[eq1[[3]] + eq2[[2]]]

$\mathrm{a} 13=0 ; \mathrm{a} 32=-\mathrm{a} 23$;

eq

conclusions from eq1,2,6:

Simplify[-2eq6[[1]] + eq1[[1]]]

Simplify[eq1[[2]] - eq2[[3]]]

$\mathrm{a} 12=0 ; \mathrm{a} 33=\mathrm{a} 22 ;$

eq

Clear[a13, a12, a32, a33]

2. case: $u=2 s \neq 0$

$u=2 s ;$ eq

conclusions from eq8, eq1:

a32 = a23; a13 = -2a23; eq

conclusions from eq3:

$\mathrm{b} 13=0 ; \mathrm{eq}$

Simplify[eq1[[2]] - eq2[[3]]]

$\mathrm{a} 12=-(\mathrm{a} 33-\mathrm{a} 22) ; \mathrm{eq}$ 
Simplify[eq3[[2]] - 1/2eq1[[1]] + 2eq1[[2]]]

a33 $=-\mathrm{a} 22 ; \mathrm{eq}$

It follows that $\mathrm{T}(\mathrm{a} 4)=0, \mathrm{~V}(\mathrm{a} 4)=-4 \mathrm{a} 22 \mathrm{a} 4, \mathrm{~W}(\mathrm{a} 4)=4 \mathrm{a} 23 \mathrm{a} 4$.

Simplify $[$ eq4 $[[2]]+$ eq2[[1]]]

Simplify $[$ eq4 $4[3]]+$ eq1[[1]]]

$\mathrm{b} 23=-\mathrm{a} 23 ; \mathrm{b} 33=-\mathrm{a} 22$;

\section{Lemma 4}

\section{Gauss for Levi-Civita connection (affine hypersphere)}

gaussLC $\left[\mathrm{x}_{-}, \mathrm{y}_{-}, \mathrm{z}_{-}\right]:=\mathrm{Na}[x, \mathrm{Na}[y, z]]-\mathrm{Na}[y, \mathrm{Na}[x, z]]-\mathrm{Na}[\mathrm{Na}[x, y]-\mathrm{Na}[y, x], z]-H h[y, z] x+$ $H h[x, z] y+K[x, K[y, z]]-K[y, K[x, z]] ;$

eq11:=Simplify[gaussLC[e[1], $e[2], e[2]]] ;$ eq12:=Simplify[gaussLC[e[1], $e[3], e[2]]] ;$

eq13:=Simplify[gaussLC[e[2], $e[3], e[2]]] ;$ eq14:=Simplify[gaussLC[e[1], $e[2], e[1]]]$;

eq15:=Simplify[gaussLC[e[1], $e[3], e[1]]] ;$ eq16:=Simplify[gaussLC[ $e[2], e[3], e[1]]] ;$

eq17:=Simplify[gaussLC[e[1], $e[2], e[3]]] ;$ eq18:=Simplify[gaussLC[e[1], $e[3], e[3]]]$;

eq19:=Simplify [gaussLC $[e[2], e[3], e[3]]]$;

eqG: $=\{$ eq11, eq12, eq13, eq14, eq15, eq16, eq17, eq18, eq19 $\}$;

\section{2. case: $u=2 s \neq 0$}

eqG

Simplify[eq11[[1]] - eq12[[3]]]

Simplify[eq15[[3]] + eq12[[3]]]

Contradiction to eq13.3

Clear[b33, b23, a33, a12, b13, a32, a13, u]

\section{1. case: $\mathrm{u}^{\wedge} \mathbf{2} \neq 4 \mathrm{~s}^{\wedge} \mathbf{2}$}

$\mathrm{a} 13=0 ; \mathrm{a} 32=-\mathrm{a} 23 ; \mathrm{a} 12=0 ; \mathrm{a} 33=\mathrm{a} 22 ; \mathrm{eqG}$

\section{Acknowledgements}

Partially supported by the DFG-Project PI 158/4-5 'Geometric Problems and Special PDEs'.

\section{References}

[1] Bryant R.L., Second order families of special Lagrangian 3-folds, in Perspectives in Riemannian Geometry, CRM Proc. Lecture Notes, Vol. 40, Amer. Math. Soc., Providence, RI, 2006, 63-98, math.DG/0007128.

[2] Calabi E., Complete affine hyperspheres. I, in Convegno di Geometria Differenziale (INDAM, Rome, 1971), Symposia Mathematica, Vol. X, Academic Press, London, 1972, 19-38.

[3] Dillen F., Equivalence theorems in affine differential geometry, Geom. Dedicata 32 (1989), 81-92.

[4] Dillen F., Katsumi N., Vrancken L., Conjugate connections and Radon's theorem in affine differential geometry, Monatsh. Math. 109 (1990), 221-235.

[5] Dillen F., Vrancken L., Calabi-type composition of affine spheres, Differential Geom. Appl. 4 (1994), 303328.

[6] Hu Z., Li H.Z., Vrancken L, Characterizations of the Calabi product of hyperbolic affine hyperspheres, Results Math. 52 (2008), 299-314.

[7] Li A.M., Simon U., Zhao G.S., Global affine differential geometry of hypersurfaces, de Gruyter Expositions in Mathematics, Vol. 11, Walter de Gruyter \& Co., Berlin, 1993. 
[8] Lu Y., Scharlach C., Affine hypersurfaces admitting a pointwise symmetry, Results Math. 48 (2005), 275300, math.DG/0510150.

[9] Nölker S., Isometric immersions of warped products, Differential Geom. Appl. 6 (1996), 1-30.

[10] Nomizu K., Sasaki T., Affine differential geometry. Geometry of affine immersions, Cambridge Tracts in Mathematics, Vol. 111, Cambridge University Press, Cambridge, 1994.

[11] O'Neill B., Semi-Riemannian geometry. With applications to relativity, Pure and Applied Mathematics, Vol. 103, Academic Press, Inc. [Harcourt Brace Jovanovich Publishers], New York, 1983.

[12] Ponge R., Reckziegel H., Twisted products in pseudo-Riemannian geometry, Geom. Dedicata 48 (1993), $15-25$.

[13] Scharlach C., Indefinite affine hyperspheres admitting a pointwise symmetry. Part 1, Beiträge Algebra Geom. 48 (2007), 469-491, math.DG/0510531.

[14] Vrancken L., Special classes of three dimensional affine hyperspheres characterized by properties of their cubic form, in Contemporary Geometry and Related Topics, World Sci. Publ., River Edge, NJ, 2004, 431459 . 